

ON COMPLEXES OF ABELIAN GROUPS WITH

APPLICATIONS TO FRACTIONAL FACTORIAL DESIGNS ${ }^{2}$

by

H. Pesotan, ${ }^{2}$ B. I. Raktoe, ${ }^{2}$ and W. T. Federer

University of Guelph and Cornell University

${ }^{1}$ Research supported by NIH Research Grant No. 5-R01-GM-05900.

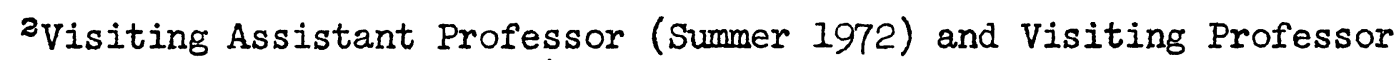
(Sept. 1, 1971 - Sept. 1, 1972), Biometrics Unit, Cornell University.

Paper No. BU-425̄M, in the Biometrics Unit Mimeo Series, Cornell University, Ithaca, New York, 14850.

Key words and phrases. Factorials, fractional factorial designs, groups, combinatorics, faithful complex, unfaithful complex, enumeration, permutation, information matrix, spectra. 


\section{ABSTRACT \\ ON COMPLEXES OF ABELIAN GROUPS WITH APPLICATIONS TO \\ FRACTIONAL FACTORIAL DESIGNS}

This paper utilizes basic group theory in formulating and resolving some fundamental algebraic problems associated with the characterization and enumeration of fractional factorial designs. Specifically it shows how the set of all possible designs based on complexes (= non-eapty subsets) of abelian groups with arbitrary replications can be divided and enumerated via faithful (i.e. complexes which under addition of elements of the abelian group produce as many complexes as the order of the group) and unfaithful (= not faithful) complexes. This is achieved using permutation theory which was in an earlier paper shown to produce classes of spectrum invariant designs with respect to an allowable set of parameters. Thus the problem of listing all possible fractional factorial designs has been reduced in this paper to listing a set of faithful and unfaithful complexes each of which produces a spectral invariant class of complexes under translation with respect to an allowable set of parameters. Finally we give formulae which count the cardinality of the set of faithful and unfaithful complexes which generate the class of all designs via replication and translation. 
ON COMPLEXES OF ABELIAN GROUPS WITH APPLICATIONS TO

FRACTIONAL FACTORIAL DESIGNS ${ }^{1}$

Running Head: COMPLEXES OF ABELIAN GROUPS

By H. Pesotan,,$^{2}$ B. L. Raktoe, ${ }^{2}$ and W. T. Federer

University of Guelph and Cornell University

1. Introduction. Although the theory of factorial and fractional factorial designs has now a history of many years, it is only recently that some of its deeper mathematical and combinatorial aspects are being exhibited and investigated. In this connection one of the earliest papers which used an abstract algebraic system to obtain results in confounding theory in the case of the symmetrical prime power factorials and hence also fractional replication is the classical paper of Fisher [1942]. Subsequently, Bose [1947] utilized finite geometrical methods to discuss and resolve certain problems in the confounding and enumeration for the prime powered symmetrical factorial. These two papers are pioneering efforts from the viewpoint of utilizing abstract mathematical systems in the study of symmetrical factorials.

\footnotetext{
'Research supported by NIH Research Grant No. 5-R01-GM-05900.

${ }^{2}$ Visiting Assistant Professor (Summer 1972) and Visiting Professor (Sept. 1, 1971 - Sept. 1, 1972), Biometrics Unit, Cornell University.

Paper No. BU-423M, in the Biometrics Unit Mimeo Series, Cornell University, Ithaca, New York, 14850.

Key words and phrases. Factorials, fractional factorial designs, groups, combinatorics, faithful complex, unfaithful complex, enumeration, permutation, information matrix, spectra.
} 
The present paper is not only in the spirit of the above mentioned two authors but also formulates the algebraic and combinatorial aspects of arbitrary fractional factorial designs from arbitrary factorials, using only the basic notions of group theory. In general, we have studied a universal class of fractional factorial designs obtained from selecting subsets of arbitrary cardinality from the group of treatment combinations with the aim to characterize and enumerate subclasses of designs such that the spectra of the underlying information matrices are the same with respest to an allowable set of parameters. In addition, we provide a method such that these subclasses of designs generate the whole universal class. The impetus for this development arose from a paper by Raktoe and Eederer [1971] and a paper by Srivasta, Raktoe and Pesotan [1971] which were preceded by the fundanental paper of Paik and Federer [1970].

In section 2 we introduce the concepts of faithful and unfaithful complexes for an anbitrary finite abelian group $G$ and study the basic properties of these complexes. In particular, we characterize unfaithful complexes as precisely those subsets of $G$ which are set unions of distinct cosets belonging to a nonzero subgroup of $\mathrm{G}$. We also introduce here the notion of length for an unfaithful complex and obtain criteria for an unfaithful complex to be of a given length. In section 3 we present recursive formulae which count the cardinality of the set of all unfaithful complexes of a given order $v$, and we use these formulae to obtain the cardinality of the smallest set of complexes of order $v$ under translation. In the paper by Raktoe and Federer [1971] the term "generator" was used for both the terms faithful and unfaithful complexes. In this 
paper we have abandoned this terminology since the term generator has a distinct meaning in group theory. In the paper by Raktoe and Federer [1971] a formula is given connecting the cardinality of all the main effect plans in the $s^{t}$ factorial, $s=p^{\alpha}, p$ a prime, in terms of the number of genera,tors which produce $s^{t}$ plans and those which do not. This formula is not true in general, though it holds for tam classes of complexes, and in section 3 we give a counter example to this effect. In addition, in tias section we give necessary and sufricient conditions under which the fahtinemeneren fomma holas. In section 4 we show how the theory develcpec in soctions 2 and 3 ma,y be applied to classify and enumerate designs. In particular, we show that the class of all designs of a chetinet crater y ray he generated by the cperation of translation followed by replicetion on a minzmal set $g$ of complexes of order $v$. In adoition, we deseribe a ganerel classification problem for designs of distinct orcer $y$ and show how the theory developed in the previous sections helps in the resolution of this problem.

\section{Charanterization and properties of faithful and unfaithful} complexes. Let $G$ be a finite additive abelian group. We will denote the cperation in $G$ by the symbol + . We call a nonempty subset $H$ of $G$ a complex and we refer to the cardinality of the set $H$ as the order of the comolex $H$. For any $g$ in $G$ we mean by $H+g$ the complex $H+g=\{h+g / h \in H\}$. In this section we introduce the concepts of faithful and unfaithful complexes as particular types of complexes of $G$. We establish here that unfeithful compleyes are precisely those comiexes of $G$ obtainable as set unions of distinct cosets of some non-zero subgroup of $G$, that is 
a subgroup distinct from the subgroup of G consisting only of the zero element of $\mathrm{G}$. As a consequence we obtain some results on the order of unfaithful complexes of $G$. In this section we also introduce the notion of length for an unfaithful complex and study the connections between a given unfaithful complex $H$ of $G$ of a given length and the complex $\mathrm{H}^{\mathrm{c}}$ which is the set complement of $\mathrm{H}$ in $\mathrm{G}$. Finally in this section we study the action of certain permutation groups of $G$ on a set of complexes of a given order for the particular case where $G$ is a finite direct product of finite additive abelian groups with the componentwise operation.

Definition 2.1. A subset $\mathrm{H}$ of $\mathrm{G}$ will be called an unfaithful subset if and only if ( $i$ ) $\mathrm{H}$ is the empty set or (ii) $\mathrm{H}$ is a complex and there exists a $g$ in $G, g \neq 0$ ( $O$ is the zero element [additive identity] of $G$ ) such that $H+g=H$. A nonempty unfaithful subset will be called an unfaithful complex. Further a complex $\mathrm{H}$ will be called a faithful complex if and only if for every $g$ in $G$ such that $g \neq 0$, we have that $H+g \neq H$.

Definition 2.2. Let $\mathrm{H}$ be any complex of $\mathrm{G}$. We say that an element $g$ in $G$ subtracts in $H$ if and only if for each $h$ in $H$ there exists $a \mathrm{k}$ in $\mathrm{H}$ with $\mathrm{h}-\mathrm{k}=\mathrm{g}$.

Proposition 2.1. A complex $\mathrm{H}$ of $\mathrm{G}$ is an unfaithful complex if and only if there exists a $g$ in $G$ different from the zero element of $G$ such that $g$ subtracts in $\mathrm{H}$.

Proof. Suppose that $\mathrm{H}$ is an unfaithful scolox. This implies that there exists a $\xi \neq 0$ such that $H+g=H$. Hence for eäch $h$ in $H$ there exists $a k$ in $\mathrm{H}$ with $\mathrm{h}=\mathrm{k}+\varepsilon$, that is, $\mathrm{h}-\mathrm{k}=\tilde{g}$ and hence 8 subtracts In $\mathrm{H}$. Conversely, suppose that $\delta \neq 0$ in $G$ subtracts in $\mathrm{H}$. We claim that $H+g=H$. Select any $h$ in $H$. Then by assumption there 
exists a $k$ in $H$ with $h-k=g$, that is, $h$ belongs to the complex $H+g$. Hence, $H \subseteq H+g$, and since the map from $H$ into $H+g$ given by $h \rightarrow h+g$ is one-to-one and onto, and since $\mathrm{H}$ is a finite set, we have that $\mathrm{H}+\mathrm{g}=\mathrm{H}$. Consequently $\mathrm{H}$ is an unfaithful complex, and this completes the proof.

The above proposition leads us immediately to:

Corollary 2.1. A complex $H$ of $G$ is a faithful complex if and only if every $B \neq 0$ in $G$ does not subtract in $\mathrm{H}$.

Let $\mathrm{H}$ be any complex in $\mathrm{G}$. Define a binary relation $\rho_{\mathrm{H}}$ in $\mathrm{G}$ by $g_{1} \rho_{H} g_{2}$ if and only if $\mathrm{H}+g_{1}=H+g_{2}$. It is easily verified that $\rho_{H}$ is a congruence relation on $G$, that is, it is an equivalence relation on $G$ such that $g_{1} \rho_{H} g_{2}$ and $f_{1} \rho_{H} f_{2}$ implies $\left(g_{1}+f_{1}\right) \rho_{H}\left(g_{2}+f_{2}\right)$ where $g_{1}, g_{2}$, $f_{1}$, and $f_{2}$ are in $G$. As a consequence it follows that the set

$$
S_{H}(G)=\left\{g / g \in G, g \rho_{H} 0\right\},
$$

where $O$ is the zero element of $G$, is a subgroup of $G$. We will refer to the subgroup $\mathrm{S}_{\mathrm{H}}(G)$ as the subgroup induced by $\mathrm{H}$, and generally, we will write $S_{H}$ for $S_{H}(G)$ when no ambiguity arises as to the group $G$ in question. We observe that if the complex $H$ contains the zero element of $G$, then $S_{H}$ is a subset of $H$ and, further, that if $H$ is an unfaithful (faithful) complex of $G$, then since $G$ is an abelian group, $H+g$ is an unfaithful (faithful) complex of $G$ for any $g$ in $G$.

Theorem 2.1. Let $\mathrm{H}$ be a complex of $\mathrm{G}$. Then $\mathrm{H}$ is an unfaithful complex if and only if $\mathrm{H}$ is a set union of distinct cosets belonging to some nonzero subgroup of $G$.

Proof. Suppose that $H$ is an unfaithful complex. Then by definition there exists a nonzero $g$ in $G$ such that $H+g=H$. Hence $g$ 
belongs to $\mathrm{S}_{\mathrm{H}}$ so that $\mathrm{S}_{\mathrm{H}}$ is a nonzero subgroup of $\mathrm{G}$. Consider the relation $\rho_{H}$ restricted to $H$ and pick $h_{1}, h_{2}, \cdots, h_{n}$ such that $h_{i} \in H, I \leq i \leq n$, is a representative from each equivalence class in the quotient set $\mathrm{H} / \rho_{\mathrm{H}}$. Then we claim that $H=\bigcup_{i=1}^{n}\left(S_{H}+h_{i}\right)$. Select any $h$ in $H$. Then, $h_{\rho_{H}} h_{i}$ for exactly one $i(I \leq i \leq n)$. This implies that $H+h=H+h_{i}$, that is, $H+\left(h-h_{i}\right)=H$. Thus $\left(h-h_{i}\right)$ belongs to $S_{H}$, and it follows that $h$ belongs to $S_{H}+h_{i}$. Hence, $H \subseteq \bigcup_{i=1}^{n}\left(S_{H}+h_{i}\right)$. Next select any $k$ in $\prod_{i=1}^{n}\left(s_{H}+h_{i}\right)$ then $k=s+h_{i}$ for some $s$ in $S_{H}$ and some $i(I \leq i \leq n)$. Hence $k$ belongs to $H+s$. But $H+s=H$ since $s \in S_{H}$, and, thus it follows that $H=\bigcup_{i=1}^{n}\left(S_{H}+h_{i}\right)$. Select $h_{i}$ and $h_{j}$ with $i \neq j(1 \leq i, j \leq n)$ and suppose that $\left(s_{H}+h_{i}\right) \cap\left(s_{H}+h_{j}\right) \neq b$. Then there exists $s$ and $t$ in $s_{H}$ with's $+h_{i}=t+h_{j}$ and thus $\left(h_{i}-h_{j}\right) \in S_{H}$. It follows that $H+h_{i}$ $=H+h_{j}$, that is, $h_{i} \rho_{H} h_{j}$ a contradiction to the choice of $h_{i}(I \leq i \leq n)$. Hence $H$ is a disjoint union of cosets belonging to the subgroup $\mathrm{S}_{\mathrm{H}}$ of $\mathrm{G}$. Conversely suppose that $H=\bigcup_{i=1}^{n}\left(s+a_{i}\right)$ is a disjoint union of cosets belonging to scme nonzero subgroup $S$ of $G$. Pick any $s$ in $S$ such that $s \neq 0$. Then since $s$ is a subgroup we have clearly that $H+s=\bigcup_{i=1}^{n}\left(s+a_{i}+s\right)$ $=\bigcup_{i=1}^{n}\left(s+a_{i}\right)=H$, that is, $H$ is an unfaithful complex and this completes the proof.

Definition 2.3. We say that an unfaithful complex $\mathrm{H}$ of $\mathrm{G}$ is based on a nonzero subgroup $S$ of $G$, or alternatively that $S$ underlies $H$ if and only if $\mathrm{H}$ is a set union of distinct cosets of $\mathrm{S}$.

Corollary 2.2. Let $S$ be any nonzero subgroup of 6 of order $s$ and index $t$. Then any unfaithful complex $H$ of $G$ based on $S$ has order ks for some $k(1 \leq k \leq t)$.

Proof. The result follows immediately from Theorem 2.1. using the fact that the order of each coset of $S$ is equal to the order of $S$. 
Theorem 2.2. Let $\mathrm{H}$ be an unfaithful complex of G. Then the subgroup $S_{H}$ induced by $H$ underlies $H$. Further, $H$ is based on a nonzero subgroup $S$ of $G$ if and only if $S$ is contained in $S_{H}$.

Proof. It is shown in the proof of Theorem 2.1. that the subgroup $\mathrm{S}_{\mathrm{H}}$ underlies $\mathrm{H}$, that is, $\mathrm{H}=\bigcup_{i=1}^{n}\left(\mathrm{~S}_{\mathrm{H}}+\mathrm{h}_{i}\right)$ a disjoint union of cosets belonging to $\mathrm{S}_{\mathrm{H}}$ - Next suppose that $\mathrm{H}$ is based on a subgroup $\mathrm{S}$ of $\mathrm{G}$. Select any $\mathrm{S}$ in $S$. Then it follows that $H+S=H$ and hence $s \in S_{H}$ by the definition of $\mathrm{S}_{\mathrm{H}}$ - Conversely, suppose that $\mathrm{S}$ is a nonzero subgroup contained in $\mathrm{S}_{\mathrm{H}}$. Then we can write $\mathrm{S}_{\mathrm{H}}$ as a disjoint union of cosets of $\mathrm{S}$, that is, $\mathrm{S}_{\mathrm{H}}$ $=\bigcup_{j=1}^{k}\left(s+a_{j}\right)$. It follows that for each $i,(I \leq i \leq n)$, the $\operatorname{coset} S_{H}+h_{i}$ $=\bigcup_{j=1}^{k}\left(s+a_{j}+h_{i}\right)$ is a disjoint union of cosets of $\mathrm{s}$. Then $\mathrm{H}$ $=\bigcup_{i=1}^{n}\left[\bigcup_{j=1}^{k}\left(s+a_{j}+h_{i}\right)\right]$ is a disjoint union of cosets of $s$, and the subgroup $\mathrm{S}$ underlies $\mathrm{H}$. This completes the proof.

Definition 2.4. Let $l$ be a natural number, $l \geq 0$. We say that an unfaithful complex $H$ of $G$ is of length $\ell$ if and only if $H$ is a disjoint union of $\ell$ cosets of some nonzero subgroup $S$ of $G$ and $H$ cannot be written as a disjoint union of $\mathrm{k}$ cosets of some nonzero subgroup of $\mathrm{G}$ with $\mathrm{k}<2$. If $\mathrm{H}$ is an unfaithful subset and $\mathrm{H}=6$ (=empty set) we define its length to be zero.

The next result gives the connection between the concept of length for an unfaithful complex $\mathrm{H}$ and the subgroup $\mathrm{S}_{\mathrm{H}}$ induced by $\mathrm{H}$.

Theorem 2.3. Let $\&$ be a positive number. The following statements are equivalent for an unfaithful complex $H$ of G:

(1) : H has length $\ell$.

(2) $\mathrm{H}$ is a disjoint union of $\&$ cosets belonging to $S_{\mathrm{H}}$. 
(3) The order of $\mathrm{H}$ is $2 \mid \mathrm{S}_{\mathrm{H}} !$, where $\mid \mathrm{S}_{\mathrm{H}} \perp$ is the order of $\mathrm{S}_{\mathrm{H}}$.

Proof. $(1) \Rightarrow(2)$. Suppose that $\mathrm{H}$ has length $\&$. Then $H$ is a disjoint union of $l$ cosets belonging to some nonzero subgroup $S$ of $G$, say $H=\bigcup_{i=1}^{\ell}\left(S+a_{i}\right)$ and there exists no representation of $H$ as a disjoint union of fewer than $l$ cosets belonging to some nonzero subgroup of $G$. However, by Theorem 2.2., the subgroup induced by $\mathrm{H}$, namely $\mathrm{S}_{\mathrm{H}}$, underlies $H$, say $H=\bigcup_{i=1}^{n}\left(s_{H}+h_{i}\right)$. It follows that $\ell \leq n$. Also by Theorem 2.2 . we have that $S \subseteq S_{H}$. Suppose $\left|S_{H}\right|=t|S|$; then the order of $H$ is on the one hand $\&|s|$ and on the other hand $n\left|s_{H}\right|=t n|s|$. It follows that $\&=t n$, and since $\ell \leq \mathrm{n}$ we must have that $\ell=\mathrm{n}$ and $t=1$. Thus $\mathrm{S}=\mathrm{S}_{\mathrm{H}}$ and (2) follows.

$(2) \Rightarrow(3)$. Statement (3) follows immediately from (2), since the order of each coset of $\mathrm{S}_{\mathrm{H}}$ is the same as the order of $\mathrm{S}_{\mathrm{H}}$.

(3) $\Rightarrow(1)$. By Theorem 2.2. we have that $H=\bigcup_{i=1}^{n}\left(S_{H}+h_{i}\right)$. Hence the order of $\mathrm{H}$ is $\mathrm{n}\left|\mathrm{S}_{\mathrm{H}}\right|$, and by (3) we then have that $\mathrm{n}=\ell$. Also if $\mathrm{H}$ $=\bigcup_{i=1}^{m}\left(s+a_{i}\right)$, that is, a disjoint union of $m$ cosets belonging to some nonzero subgroup $S \neq S_{H}$, then by Theorem 2.2., $S$ is strictly contained in $\mathrm{S}_{\mathrm{H}}$ so that $|\mathrm{s}|<\left|\mathrm{S}_{\mathrm{H}}\right|$. It follows that $\ell<\mathrm{m}$, so that $\mathrm{H}$ has length $\ell$ and this completes the proof.

The following is now an immediate consequence of Theorem 2.2. and Theorem $2 \cdot 3$.

Corollary 2.3. Each unfaithful subset $\mathrm{H}$ of $\mathrm{G}$ has an unique length . If $\mathrm{H}=b$ then $l=0$ and if $\mathrm{H} \neq b$ the number $l$ is the number of distinct cosets of $\mathrm{S}_{\mathrm{H}}$ whose union is $\mathrm{H}$. 
Theorem 2.4. Let $\mathrm{G}$ have order $\mathrm{n}$ and let $v$ be a number such that $I \leq \nu \leq n$. Then there exists an unfaithful complex of order $v$ if and only if the $\mathrm{g} \cdot \underline{c} \cdot \mathrm{d} \cdot(x, \mathrm{n})>1$.

Proof. Suppose that $H$ is an unfaithful complex of order $v$ and length \&. Then, by Theorem 2.3., $v=\ell\left|S_{H}\right|$ where $\left|S_{H}\right|>1$, and since $\left|S_{H}\right|$ divides $\mathrm{n}$, we have that the g. c. d. between $v$ and $\mathrm{n}$ is larger than 1 . Next suppose that the g. c. d. $(v, n)>1$. Let the g. c. d. $(v, n)=d>1$ and say that $v=d k$. Let $S$ be any subgroup of $G$ of order $d$, then since $d>1$, $S$ is a nonzero subgroup. Let $\mathrm{H}$ be a set union of $\mathrm{k}$ distinct cosets of $\mathrm{S}$. Then, by Theorem 2.1., $\mathrm{H}$ is an unfaithful complex and $\mathrm{H}$ has order $\nu$ by construction. This completes the proof.

Proposition 2.2. If $\mathrm{H}$ is an unfaithful complex of order $v$ and $g . \underline{c}$. $(v, n)=d$, then the order of any underlying subgroup of $H$ divides $d$ where $\mathrm{n}$ is the order of $\mathrm{G}$.

Proof. Suppose that $H$ has order $\nu$ and length $\ell$. Then, by Theorem 2.3., we have that $\nu=\ell\left|S_{H}\right|$. If $S$ is any underlying subgroup of $H$, then by Theorem 2.2. $\mathrm{S} \subseteq \mathrm{S}_{\mathrm{H}}$, so that the order of $\mathrm{S}$ divides $\left|\mathrm{S}_{\mathrm{H}}\right|$. But $\left|\mathrm{S}_{\mathrm{H}}\right|$ divides $n$, and hence $|S|$ divides $v$ and $n$. It follows that the order of $s$ divides $d$; and this completes the proof.

Let $G$ have order $n$ and let $\nu$ be any number such that $I \leq v \leq n$. Suppose that the g. c. d. $(v, n)=d>1$. Then $v=k d$ where g. c. d. $(k, n)$ = 1. Proposition 2.2. leads us to the consideration of the following sets. Let

$$
D=\left\{a_{1}, a_{2}, \cdots, a_{t}\right\}
$$


where each $d_{i}(1 \leq i \leq t)$ is a divisor of $d$ and $d_{i}>1$ for each $i$. We assume that $a_{t}=d$ and that $d_{1}<d_{2}<\cdots<d_{t}$. Further, let $d=m_{i} d_{i}$ $(I \leq i \leq t)$ and let

$$
I=\left\{k m_{i}: \quad v=k d ; I \leq i \leq t\right\}
$$

In terms of the above notation we now establish the following result.

Proposition 2.3. If $\mathrm{H}$ is an unfaithful complex of order $\nu$ and length $\ell$, then $\&$ belongs to $L$.

Proof. By Proposition 2.2. the order of the subgroup $\mathrm{S}_{\mathrm{H}}$ divides $\mathrm{d}$. Hence for some $i,(I \leq i \leq t), d_{i}=\left|s_{H}\right|$. Then $v=k d=k\left(m_{i} d_{i}\right)=\left(k m_{i}\right)$. $\left|\mathrm{S}_{\mathrm{H}}\right|$. It follows from Theorem 2.3. that $\mathrm{H}$ has length $\ell=\mathrm{km}_{i}$ so that $\ell$ belongs to $L$. This completes the proof.

Remark. We have seen in Proposition 2.3. that the length of every unfaithful complex of order $v$ is an element of $I$. However, it is not true in general that corresponding to each $\ell$ in $I$ there exists an unfaithful complex $\mathrm{H}$ of $\mathrm{G}$ of order $v$ and length $\ell$. For example, let $\mathrm{C}=\{0,1\}$ be the cyclic group of order two under addition mod. 2 and let $G=\underset{i=1}{x} C_{i}$, where $C_{i}=C(I \leq i \leq 4)$, be the direct product under the componentwise operation. Let $v=8$ so that the g. c. $d \cdot(v, n)=d=8$ as well, and thus in this case $L$ consists of all divisors of eight different from 1 . Since two divides eight, it follows that two is an element of $I$. However, there exists no unfaithful complex of $G$ of order eight and length two. For such an unfaithful complex must have an underlying subgroup of order four. Now if $S$ is a subgroup of $G$ of order four, then, in this case for any $a \in G-S$, we have that $H=S U(S+a)$ is a subgroup of order eight as can be 
readily verified. For this case, any unfaithful complex of order eight which has an underlying subgroup of order four must have length 1 .

Let $\nu$ and $l$ be any numbers such that $0 \leq v, l \leq n$ where $n$ is the order of the group $G$. Let $S$ be any nonzero subgroup of $G$. We now introduce the following sets.

(2.4) Let $F_{v}(G)$ be the set of all faithful complexes of order $v$.

(2.5) Let $U_{v}(G)$ be the set of all unfaithful subsets of order $v$.

(2.6) Let $C_{v}(G)$ be the set of all subsets of $G$ of cardinality $v$.

(2.7) Let $x_{\ell, v}(S)$ be the set of all unfaithful subsets of length $\ell$ and order $v$ which have underlying subgroup $S$.

It is clear that $C_{\nu}(G)=F_{v}(G) \cup U_{v}(G)$ and further that for each $v$ $(0 \leq v \leq n)$ this union is disjoint. Note that $x_{0, v}(s)$ and $X_{\ell, 0}(s)$ are empty sets if $\nu$ and $l$ are respectively nonzero numbers and that $x_{0,0}(S)$ is a nonempty set and equals $\{\phi\}$.

Proposition 2.4. Let $G$ have order $n$. let $S$ be a subgroup of $G$, and let $H$ be an unfaithful subset of $f$ of length $\&$ and order $v$. (i) If $H=\phi$, then the set complement $H^{c}$ in $r$ is an unfaithful complex of length $l$. (ii) If $\mathrm{H} \neq \phi$, let $\mathrm{T}$ be the subgroup induced by $\mathrm{H}$ and let $t$ be its index. Then $\mathrm{H} \in \mathrm{X}_{\ell, \nu}(S)$ if and only if $\mathrm{H}^{\mathrm{C}} \in \mathrm{X}_{t-\ell, n-\nu}(S)$. In particular, $\mathrm{T}$ is also the subgroup induced by $H^{c}$ and the map $\varphi$ given by $\varphi(H)=H^{c}$ establishes a one-to-one correspondence between the sets $U_{v}(G)$ and $U_{n-v}(G)$ such that $\infty$ carries the set $X_{\ell, v}(T)$ onto the set $X_{t-l, n-v}(T)$.

Proof. Part ( $i$ ) is obvious. Suppose that $H \neq \phi$ and that $S$ is a 
subgroup which underlies $H$. Since $G$ is a disjoint union of cosets belonging to $\mathrm{S}$, it follows immediately that $\mathrm{H}^{\mathrm{C}}$ is a disjoint union of esets belonging to $S$ so that $S$ underlies $H^{C}$ as well. Suppose next that $T_{I}$ is the subgroup induced by $\mathrm{H}^{\mathrm{C}}$. Then $\mathrm{T}_{1}$ underlies $\mathrm{H}^{\mathrm{C}}$ and it follows that $\mathrm{T}_{1}$ underlies H. But by Theorem 2.2. we must have that $\mathrm{T}_{1} \subseteq \mathrm{T}$. Conversely, since $T$ also underlies $\mathrm{H}^{\mathrm{C}}$, we have by Theorem 2.2. that $\mathrm{T} \subseteq \mathrm{T}_{1}$, resulting in $T=T_{1}$; the order of $H^{c}$ is $n-v=(t-l)|T|=(t-l)\left|T_{1}\right|$, and it follows from Theorem 2.3. that $\mathrm{H}^{c}$ has length $(t-l)$. Hence $\mathrm{H} \in \mathrm{X}_{\ell, \nu}(\mathrm{S})$ if and only if $H^{c} \in X_{t-l, v}(S)$, and $H$ and $H^{c}$ have the same induced subgroup. It is now clear that the map $\varphi$ is a one-to-one correspondence of the indicated type and this completes the proof.

The following is now an immediate corollary of the previaus proposition.

Corollary 2.4. Let $\mathrm{H}$ be a faithful complex of order $*$ in $G$ and let $G$ have order $n$ where $I \leq v \leq n$. Then, the set complement $H^{c}$ in $G$ is a faithful complex of order $(n-\nu)$ and the map $\oplus$ given by a one-to-one correspondence between the sets $F_{0}(G)$ and $F_{n-\nu}(G)$.

Proposition 2.5. Let $G$ have order $n$. Then $H$ is a faithful complex of order $v$ in G if and only if (i) the g.c.d. $(v, n)=1$ or (ii) the g.c. $d \cdot(v, n)=d>1$ and for any nonzero subgroup $S$ of $G$ there exists $\mathrm{b}$ in $\mathrm{H}$ such that $\mathrm{S}+\mathrm{b} \nsubseteq \mathrm{H}$.

Proof. Suppose that $\mathrm{H}$ is a faithful complex of order $v$ and that the g. c. $d \cdot(v, n)=d>1$. If there exists a nonzero subgroup $S$ of $G$ such that $S+b \subseteq H$ for each $b$ in $H$, then clearly $H=U\{S+a / a \in H\}$ and it follows that $\mathrm{H}$ is an unfaithful complex which is a contradiction. On the other 
hand suppose that the g. c. d. $(v, n)=1$. Then by Theorem 2.4. every complex of order $\nu$ is faithful. Finally, if the g. c. $d \cdot(\nu, n)=d>I$ and the condition stated in the proposition holds for $\mathrm{H}$, then $\mathrm{H}$ cannot have a nonzero underlying subgroup. It follows by Theorem 2.1. that $\mathrm{H}$ must be a faithful complex and this completes the proof.

For any $g$ in $G$ we define a permutation $\omega_{g}$ on the underlying set of $G$ by $\omega_{g}(x)=x+g$. Let

$$
\tilde{\tilde{C}}(G)=\left\{\omega_{\mathrm{g}} / g \in G\right\}
$$

Clearly $\tilde{\Omega}(G)$ is a subgroup of the symmetric group of all permutations of $G$ and is isomorphic to $G$ under the map $g \rightarrow \omega_{g}$.

Let $G_{1}, G_{2}, \cdots, G_{m}$ be finite additive abelian groups of orders $s_{1}$, $s_{2}, \cdots, s_{m}$ respectively and let $s=\prod_{i=1}^{m} s_{i}$. Let $K=\prod_{i=1}^{m} G_{i}$ be their direct product under the componentwise operation. For each $i$, ( $I \leqslant i \leq m)$, let $\Omega\left(G_{i}\right)$ be the symmetric group of order $s_{i}$ : on the underlying set of $G_{i}$ and let

$$
\Omega(K)=\underset{i=1}{m} \Omega\left(G_{i}\right)=\left\{\left(\omega_{1}, \cdots, w_{m}\right) / \omega_{i} \in \Omega\left(G_{i}\right)\right\}
$$

be the direct product of the groups $\Omega\left(G_{i}\right)$ under the componentwise operation of functional composition. For any $a=\left(a_{1}, \cdots, a_{m}\right)$ in $K$ and $\omega=\left(\omega_{1}, \cdots, \omega_{m}\right)$ in $\Omega(K)$, we define $\omega(a)=\left(\omega_{1}\left(a_{1}\right), \cdots, \omega_{m}\left(a_{m}\right)\right)$ and for any complex $H$ of $K$ we define $\omega(H)=\{\omega(h) / h \in H\}$. Then for any $g=\left(g_{1}, \cdots, g_{m}\right)$ in $K$ the permutation $\omega_{g}$ in $\tilde{\Omega}(K)$ is given by $\omega_{g}(a)=a+g=\left(a_{1}+g_{1}, \cdots, a_{m}+g_{m}\right)$, and clearly $\tilde{\Omega}(K)$ is a subgroup of $\Omega(K)$. For any $\alpha$ in $\Omega(K)$ we denote by $\tilde{\Omega}(K) \alpha$ a right coset of $\tilde{\Omega}(K)$ in $\Omega(K)$ determined by $\alpha$. 
Let $\Omega_{I}$ be any nonempty subset of $\Omega(K)$ and $\&$ a set of complexes of $K$ of a given order $v(I \leq \nu \leq s)$. Let

$$
\text { (2.10) } \Omega_{1} * g=\left\{\alpha(H) / \alpha \in \Omega_{1} \text { and } H \in \beta\right\} \text {. }
$$

If $\&=\{H\}$, we write $\Omega_{1} * H$ instead of $\Omega_{1} * \&$.

Definition 2.5. We call the set $\Omega_{1} * \xi$ the set generated by the action of $\Omega_{1}$ on the set of complexes $\&$. If $\Omega_{1}=\Omega(K)$ and $s=\{H\}$, we say that any two sets in $\Omega(K) *_{H}$ are related by a permutation, and if $\Omega_{I}=\tilde{\Omega}(K) \alpha$ with $\alpha \in \Omega(K)$, then we say that any two sets in $\tilde{\Omega}(K) \alpha * \mathrm{H}$ are related by a translation.

$$
\text { Given } \mathrm{H} \in \mathrm{C}_{v}(K),(I \leq v \leq s) \text {, we now ask what are the sets } \& \subseteq \mathrm{C}_{\nu}(K)
$$
which have the following two properties: (i) $\tilde{\Omega}(\mathrm{K}) * \xi=\Omega(\mathrm{K}) *_{\mathrm{H}}$ and (ii) if $\mathrm{J}$ is any set such that $I \subseteq C_{V}(K)$ with $\tilde{\Omega}(K) * \mathcal{J}=\tilde{\Omega}(K) * \&$, then $|\&| \leqslant|J|$, that is, what are the sets $\& \subseteq C_{\nu}(K)$ of least cardinality such that the class generated by the action of $\Omega(K)$ on $H$ is the same as the class generated by the action of $\tilde{\Omega}(\mathrm{K})$ on $\&$ ?

Let $R=\left\{\alpha_{1}, \cdots, \alpha_{r}\right\}$ be a set of representatives of right cosets of the subgroup $\tilde{\Omega}(K)$ in $\Omega(G)$. For $H$ in $C_{v}(K)$ and $\alpha_{i}, \alpha_{j}$ in $R$ define a relation $\sigma_{H}$ on $R$ by

$$
\begin{aligned}
& \alpha_{i} \sigma_{H} \alpha_{j} \text { if and only if there exists a } g \text { in } K \text { such that } \\
& \omega_{g}\left(\alpha_{i}(H)\right)=\alpha_{j}(H) .
\end{aligned}
$$

It is clear that $\sigma_{H}$ is an equivalence relation on $R$. In the following theorem using the above notation we answer the question posed above.

Theorem 2.5. (i) For any $\alpha$ in $\Omega(K)$ and $H$ in $C_{v}(K)$ the cardinality of the class generated by the action of the right coset $\tilde{\Omega}(K) \alpha$ on the complex $H$ 
is the index of the subgroup $S_{\alpha(H)}(K)$, induced by $H$, in $K$. (ii) Let \&. $=\left\{B_{l}(H), \cdots, B_{k}(H)\right\}$ where $k$ is the cardinality of the quotient set $R / \pi_{H}$ and $\left\{\beta_{1}, \cdots, \beta_{r}\right\}$ is a set of representatives, one form each equivalence class in $R / \sigma_{H}$. Then $(a) \Omega(K) * H=U\left\{\tilde{\Omega}(K) *_{i}(H) \mid I \leq i \leq k\right\}$ and this union is disjoint and (b) If $\mathrm{T}$ is any set of complexes contained in $\mathrm{C}_{\nu}(\mathrm{K})$ such that $ح(K) * H=\tilde{\Omega}(K) * \tau$, then $|s| s|\tau|$.

Proof. (i) Now by definition $\tilde{\Omega}(\mathrm{K}) \alpha * \mathrm{H}=\left\{w_{\mathrm{g}}(\alpha(\mathrm{H})) \mid \mathrm{g} \in \mathrm{K}\right\}$. Hence $\omega_{g_{1}}(\alpha(H))=\omega_{g_{2}}(\alpha(H))$ if and only if $\alpha(H)+g_{1}=\alpha(H)+g_{2}$, that is, if and only if $g_{1}-g_{2}$ belongs to $s_{\alpha(H)}(K)$. It follows that the cardinality of the set of complexes which are related by a translation to $\mathrm{H}$ is the index of the subgroup $S_{\alpha(H)}(K)$ in $K$, that is $\mid \tilde{\Omega}_{(K)}\left(\alpha^{*} H|=|^{K} / S_{\alpha(H)}(K) !\right.$ (ii) Pick any $\mathrm{H}_{1}$ in $\Omega(\mathrm{K}) * \mathrm{H}$. This implies that there exists an $\alpha$ in $\Omega(\mathrm{K})$ such that $H_{1}=\alpha(H)$. Now $\Omega(K)=\bigcup_{i=1}^{r} \tilde{\Omega}(K) \alpha_{i}$ and hence there exists $i$, ( $\left.I \leq i \leq r\right)$, and some $g \in \mathrm{K}$ such that $\alpha=\omega_{\mathrm{g}} \alpha_{i}$. Thus $\mathrm{H}_{1}=\omega_{\mathrm{g}} \alpha_{i}(\mathrm{~F})$. Now $\alpha_{i} \in \mathrm{R}$ implies $\alpha_{i} \sigma_{H} \beta_{j}$ for some $j(I \leq j \leq k)$, that is, $\alpha_{i}(H)=\omega_{g_{I}}\left(\beta_{j}(H)\right)$ for some $g_{I}$ in $K$. Hence $H_{I}=\alpha(H)=\omega_{g}+g_{I}\left(\beta_{j}(H)\right) \in \tilde{\Omega}(K) * \beta_{j}(H)$. It follows that $\Omega(K) * H \subseteq \tilde{\Omega}(K) * ?$ and since the reverse inclusion is clear, we have equality. Further suppose that for some $i \neq j(I \leq i, j \leq k)$, we have $\left[\tilde{\Omega}(K) *_{i}(H)\right]$ $\cap\left[\tilde{\Omega}(K) * \beta_{j}(H)\right] \neq \phi$. Then for some $g_{1}, g_{2}$ in $K$ we have that $\omega_{g_{1}}\left(\beta_{i}(H)\right)$ $=\omega_{g_{2}}\left(\beta_{j}\left(H_{j}\right)\right)$. This immediately implies that $\beta_{i} \sigma_{H} \beta_{j}$ and this contradicts the choice of the $\beta_{i}(I \leq i \leq k)$. Hence $\Omega(K)$ * $\mathrm{H}$ is a disjoint union of the sets $\left[\tilde{\Omega}(K) * \beta_{i}(H)\right]$ where $(I \leq i \leq k)$. Next suppose that $\Omega(K) * H=\tilde{\Omega}(K) * J$ for some $I \subseteq C_{v}(K)$. For $\beta_{i}(H) \epsilon \&$, where $I \leq i \leq k, B_{i}(H)=\alpha(H)$ for some $\alpha$ in $\Omega(K)$, and hence $\beta_{i}(H)=\omega_{g_{I}}\left(H_{i}\right)$ for some $H_{i}$ in $J$ and $g_{I} \in K$. Consider the map $\varphi$ from \& into $T$ which assigns to each $\beta_{i}(H)$ in \& the complex $H_{i}$ in I such that $\beta_{i}(H)=\omega_{g_{I}}\left(H_{i}\right)$ for some $g_{I}$ in $K$. Suppose that $\varphi\left(\beta_{i}(H)\right)=\varphi\left(\beta_{j}(H)\right)$. 
Then for some $g_{1}, g_{2}$ in $K$ we have that $\beta_{i}(H)=\omega_{g_{1}}\left(H_{i}\right)=\omega_{g_{2}}\left(H_{j}\right)=\beta_{j}(H)$. This immediately implies that $\beta_{i} a_{H} \beta_{j}$ and hence by the choice of the $\beta_{r}^{\prime} s$ we must have that $\beta_{i}=\beta_{j}$. Hence the map $\varphi$ is one-to-one and $|s| s|\tau|$. This completes the proof.

The following example illustrates some of the ideas in this section. It shows also that, in general, under permutations from the group $\Omega(K)$ the properties of faithfulness, unfaithfulness and length for a complex $H$ of $K$ need not be preserved.

Example. Iet $C_{4}=\{0,1,2,3\}$ be the four element cyclic group under addition mod. 4. Let $\mathrm{K}=\mathrm{C}_{4} \times \mathrm{C}_{4}$ be tire direat product of $\mathrm{C}_{4}$ with itseli under the componentwise reration, Iet $(i) A=\{(0,0),(1,2),(2,0),(3,2)\}$ (ii) $A_{1}=\{(c, 0),(2,2),(1,0),(3,2)\}$ and $($ iii $) A_{2}=\{(0,0),(1,3),(2,0),(3,3)\}$.

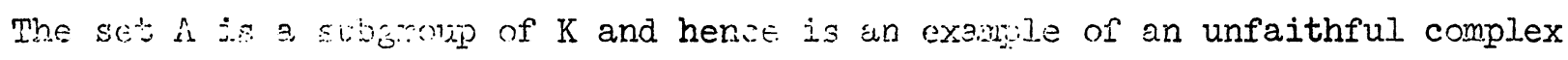
of length 1. Cn the other hand, the sets $A_{1}$ and $A_{2}$ are not subgroups of $\mathrm{K}$ but ane wajons of cosets belonging to the singrons $\{(0,0),(2,2)\}$ and $\{(0,0),(2,0)\}$ restectively. Hency by reo:en 2.l. $A_{1}$ and $A_{2}$ are unfathful complexes of $\mathrm{K}$ of order 4 . Furting, ean of the unfaithful complexes $A_{1}$ and $A_{2}$ have Iersolin 2 and the sugroup induesd by $A_{1}$ is $\{(0,0),(2,2)\}$ whilst the subgroup incus a $A_{2}$ is $\{(0,0),(2,0)\}$. Consicien the permu-

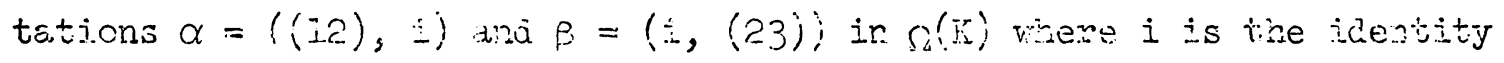
permutation on $C_{4}$ and $(i 2),(23)$ ara two cyoies on $C_{4}$. Then $\alpha(A)=A_{1}$ and $\beta(A)=A_{2}$ so thei, $\alpha, \beta$ preserve the property of metitrfulness of $A$ in this

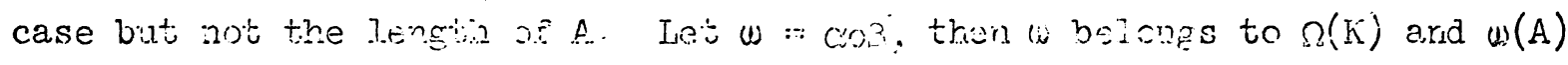
$=\{(0,0),(2,3),(1,0),(3,3)$ ? winch is a fuifreul conlex of order 4 . Hence $\omega$ carries an unatihful comple: into a faithou coplex shoring that, in general, permutations in the gioup $\Omega(K)$ need not preserve the property of 
faithfulness or unfaithfulness.

3. On counting the number of Paithful and unfaithful complexes.

Throughout this section the following notation will be used: (i) We will denote the order of the abelian group $G$ by $n$ and $\nu$ will be a fixed number such that $I \leq v \leq n$. If $X$ is any set, we will denote the cardinality of $X$ by $|x|$. (ii) For any number $q, I \leq q \leq n$, such that $q$ divides $n$, let $K_{q}(G)$ denote the set of all subgroups of $G$ of order $q$. When it is clear from the context which abelian group $G$ is being considered, we shall write $\mathrm{K}_{\mathrm{q}}$ for $K_{q}(G)$. For any nurbers $q_{1}$, $q_{2}$ such that $1 \leq q_{1}<q_{2} \leq n$ and for a given subgroup $T \in K_{q_{2}}$ let $R\left(K_{q_{1}}, T\right)=\left\{S / S \in K_{q_{1}}\right.$ and $S$ is a subgroup of $\left.T\right\}$, if $q_{I}$ divides $c_{2}$, erd, let $R\left(K_{q_{I}}, T\right)=\phi$ otherwise. Further, let $\psi_{G}(q)$ $=\left|K_{q}\right|$ and let $\psi_{T_{x}}\left({ }_{1}, \cdots\right)=\left|R\left(K_{q_{1}}, T\right)\right|$. Note that $\psi_{G}\left(q_{1}, T\right)=0$ if $q_{1}$ does not divide $q_{2}$. (ij门) Tun any numbers $s$ and $\ell,(I \leq s, l \leq n)$, we define the set $X_{\ell, v}\left(K_{S}\right)=\left\{H / H \in U_{v}(G), H\right.$ has length $\ell$ and an underlying subgroup $\mathrm{S} \in \mathrm{K}_{\mathrm{S}}$ \}, that is, the set of all unfaithful complexes of order $\nu$ and length $b$ which have an underlying subgroup of order s. Since the number $v$ is fixed throughout this soction we will write $X_{\ell}\left(K_{s}\right)$ for $X_{2, \nu}\left(K_{s}\right)$. Sililarly ve write $x_{\ell}(s)$ for the set $x_{2, v}(s)$ defined by $(2.7)$.

We introduce a binary relation $T$ in the set of complexes of order $*$, namely in $C_{v}(G)$, as follows:

$\mathrm{H}_{1} \tau \mathrm{H}_{2}$ if and only if there exists a $\mathrm{g}$ in $\mathrm{G}$ such that $\omega_{\mathrm{g}}\left(\mathrm{H}_{1}\right)$ $=\mathrm{H}_{2}$ where $\omega_{\mathrm{g}}$ is the mapping introduced in (2.8).

It is clear that $\tau$ is an equivalence relation on $C_{V}(G)$. In this section we will consider restrictions of $\tau$ to various subsets of $C_{v}(G)$. When no confusion is likely as to the group $G$ in question we will write $F_{v}, U_{v}$, and $C_{v}$ 
respectively for the sets $F_{v}(G), U_{v}(G), C_{v}(G)$ and we will denote the corresponding quotient sets by $F_{v} / \tau, U_{v} / \tau$, and $C_{v} / \tau$ respectively.

We denote an equivalence class under $\tau$ by $[H]_{\tau}$. Then, we have clearly that

$$
\tilde{\Omega}(G) * \mathrm{H}=[\mathrm{H}]_{\tau},
$$

that is, the class $[\mathrm{H}]_{\tau}$ consists of complexes of order $\nu$ which are related by translation to $\mathrm{H}$, indeed, $[\mathrm{H}]_{\tau}=\left\{\mathrm{H}^{\prime} / \mathrm{H}^{\prime} \in \mathrm{C}_{\nu}, \mathrm{H}^{\prime}=\mathrm{H}+\mathrm{g}, \mathrm{g \in G}\right\}$. Further, it is clear that $[H]_{\tau}$ belongs to exactly one of $F_{\nu / \tau}$ or $U_{\nu} / \tau$ according as $H$ belongs to $F_{v}$ or $U_{v}$ respectively.

Let $\&=\left\{\mathrm{H}_{1}, \mathrm{H}_{2}, \cdots, \mathrm{H}_{\mathrm{c}}\right\}$ be a set of representatives one from each equivalence class of the quotient set $C_{\nu} / \tau$. Then, clearly, \& is the set with the smallest cardinality such that $\tilde{\Omega}(G) * C_{\nu}=C_{\nu}(G)$, that is, (a) $C_{\nu}(G)$ $=\left\{H_{i}+g / I \leq i \leq c, g \in G\right\}$ and $(b)$ if $\tilde{\Omega}(G) * J=C_{v}(G)$ for some set $J \subseteq C_{v}(G)$ then $|\&| \leq|\tau|$.

The main objectives in this section are the following:

(1) We provide recursive formulae which give the cardinality of the set of unfaithful complexes of order $v$ which have a prescribed length and underlying subgroup. In general these formulae require knowing the cardinalities of the sets of all subgroups of $\mathrm{G}$ of a given order containing, respectively contained in, a fixed subgroup of $G$.

(2) In the special case such that (i) $G$ is a cyclic group or (ii) $G$ is a direct product, $G=\left(c_{p_{2}} \times \cdots * C_{p_{1}}\right) \times\left(c_{p_{2}} \times \cdots \times c_{p_{\dot{g}}}\right) \times \cdots \times\left(C_{p_{m}} \times \cdots \times c_{p_{m}}\right)$ where $c_{p_{1}},(I \leq i \leq m)$, is a cyclic group of order $p_{i}$ and $p_{1}, p_{2}, \cdots, p_{m}$ are distinct primes we show that the formulae mentioned in (I) reduce to 
recursive formulae which allow one to calculate the cardinality $c$ of the quotient set $\mathrm{C}_{V / \tau}$. Hence we have a method in the case $G$ has the particular forms indicated to compute the cardinality of the set $\&$ of complexes satisfying the properties (a) and (b) mentioned above. In the general case, that is, for an arbitrary abelian group $G$, the cardinality $c$ of the quotient set $\mathrm{C}_{v / \tau}$ could be similarly computed if the cardinalities of the sets of subgroups of a given order containing, respectively contained in, a fixed subgroup of G are known.

(3) We state a formula given in Raktoe and Federer [1971] which connects the cardinalities of the sets $U_{V}(G) / \tau, F_{\nu}(G) / \tau$, and $C_{\nu}(G) / \tau$ for the special case where $G$ is a direct product, $G=C_{S_{2}} \times \cdots \times C_{S_{t}}$ where for each $i$, $(1 \leq i \leq t), s_{i}=p^{\alpha}, p$ is a fixed prime and $c_{s_{1}}$ a cyclic group of order $s_{i}$ and $v=t\left(p^{\alpha}-1\right)+1$. We show by an example that, in general, the $R-F$ formula is false. We then provide necessary and sufficient conditions under which the R-F formula holds.

We begin by providing the basic setting in which the results of this section will be formulated.

Suppose that the g. c. d. $(v, n)=1$ and let $D=\left\{d_{1}, d_{2}, \cdots, d_{t}\right\}$, $I=\left\{k m_{i}: I \leq i \leq t\right\}$ be the sets introduced in (2.2) and (2.3). Recall that $1<d_{1}<d_{2}<\cdots<d_{t}$, and $d_{t}=d$, and $d=m_{i} d_{i}$ for each $i(1 \leq i \leq t)$. Set $b_{i}=k m_{i}$, $(1 \leq i \leq t)$. Then by Proposition 2.3. the various possible lengths for unfaithful complexes of order $v$ occur amongst the set $\left\{b_{1}, b_{2}, \cdots, b_{t}\right\}$. Let $r_{i}=\frac{n}{a_{1}},(1 \leq i \leq t)$, and set $r_{t}=r, b_{t}=b$. Note that $b_{1}>b_{2}>\cdots>b_{t}$.

Lemma 3.1. For each $i,(1 \leq i \leq t)$, we have the following: (a) The 
set $X_{b_{1}}(S)$ is precisely the set of all unfaithful complexes of order $v$ and length $b_{i}$ which have induced subgroup $S$ if and only if the order of $S$ is $d_{i}$. (b) The set $\mathrm{X}_{\mathrm{b}_{1}}\left(\mathrm{~K}_{\mathrm{d}_{1}}\right)=U\left\{\mathrm{X}_{\mathrm{b}_{1}}(\mathrm{~s}) / \mathrm{S} \in \mathrm{K}_{\mathrm{d}_{1}}\right\}$ and this set union is pairwise disjoint. In particular the set $\mathrm{X}_{\mathrm{b}_{1}}\left(\mathrm{~K}_{\mathrm{d}_{1}}\right)$ is precisely the set of all unfaithful complexes of order $v$ and length $b_{i}$ which have an induced subgroup of order $d_{i}$. (c) If $H \in \mathrm{X}_{\mathrm{b}_{1}}(\mathrm{~S})$, where $\mathrm{S}$ is some nonzero subgroup of $\mathrm{G}$, then $[\mathrm{H}]_{\tau}$ $\subseteq \mathrm{x}_{\mathrm{b}_{1}}(\mathrm{~s})$

Proof. (a) Assume that $S$ is the induced subgroup for any unfaithful complex $H \in \mathrm{X}_{\mathrm{b}_{1}}(\mathrm{~S})$. Since $\mathrm{H}$ has length $\mathrm{b}_{i}$, we have by Theorem 2.3. that $|H|$ $=b_{i}|s|$. It follows that $|s|=d_{i}$ since $v=|H|=b_{i} d_{i}$. On the other hand suppose that $|s|=d_{i}$. Choose any $H \in \mathrm{X}_{\mathrm{b}_{1}}(\mathrm{~s})$. Then $H$ has length $\mathrm{b}_{i}$ and underlying subgroup $S$. This implies that $|H|=\ell|S|=l d_{i}$ for a suitable number $l$. But $l=b_{i}$ and $|H|=b_{i} d_{i}$. Hence, by Theorem 2.3. S is the induced subgroup of $\mathrm{H}$. (b) Select $\mathrm{H} \in \mathrm{X}_{\mathrm{b}_{1}}\left(\mathrm{~K}_{\mathrm{d}_{1}}\right)$. Then the unfaithful complex $H$ has length $b_{i}$ and an underlying subgroup $S$ of order $d_{i}$. But by part (a), $S$ is the induced subgroup for $\mathrm{H}$ and hence $\mathrm{X}_{\mathrm{b}_{1}}\left(\mathrm{~K}_{\mathrm{d}_{1}}\right)$ consists precisely of all unfaithful complexes of length $b_{i}$ which have induced subgroup of order $d_{i}$. The set equality mentioned in part (b) of the Lemma is clear. We now check to see if the union is disjoint. Suppose that $S$ and $T$ are distinct subgroups of order $d_{i}$ and that $H \in\left(x_{b_{i}}(S) \cap X_{b_{i}}(T)\right)$. Then it follows from part (a) that $S$ and $T$ are both induced subgroups of $H$. By Theorem 2.2. this means that $S=T$ a contradiction to the choice of $S$ and $T \cdot$ (c) Select $\mathrm{H} \in \mathrm{X}_{\mathrm{b}_{1}}(\mathrm{~s})$. Then there exist elements $s_{1}, s_{2}, \cdots, s_{b_{1}}$ in $\mathrm{s}$ such that $H=\left(S+s_{1}\right) \cup \cdots \cup\left(S+s_{b_{1}}\right)$, a disjoint union. But for any $g$ in $G$ we have that $\mathrm{H}+\mathrm{g}=\left(\mathrm{s}+\mathrm{s}_{1}+\mathrm{g}\right) \cup \cdots U\left(\mathrm{~s}+\mathrm{s}_{\mathrm{b}_{1}}+\mathrm{g}\right)$ which is again a disjoint union. Hence $(H+g) \in X_{b_{1}}(S)$ and thus $[H]_{\tau} \subseteq X_{b_{1}}(S)$. This completes the proof. 
Lemma 3.2. The set of all unfaithful complexes of order $v$, namely, $U_{v}=U\left\{\mathrm{X}_{\mathrm{b}_{1}}\left(\mathrm{~K}_{\mathrm{d}_{1}}\right) \mid I \leq i \leq t\right\}$ and this set union is pairwise disjoint.

Proof: Suppose that $\mathrm{H}_{\mathrm{U}} \mathrm{U}_{v}$. By Corollary 2.3. $\mathrm{H}$ has a unique length, say $l$. From Proposition 2.3. it follows that $b=b_{i}$ for exactly one $i$ $(I \leq i \leq t)$. Hence $H \in X_{b_{1}}\left(K_{d_{1}}\right)$. Thus the set equality is established. Moreover, $x_{b_{1}}\left(K_{d_{1}}\right) \cap x_{b_{j}}\left(K_{d_{j}}\right)=\phi$ for $i \neq j$, since then $b_{i} \neq b_{j}$, so that if $\mathrm{H}$ lies in the intersection $\mathrm{H}$ would have two different lengths contradicting Corollary 2.3. This completes the proof.

Lemma 3.3. For each $i,(1 \leq i \leq t)$, and a given subgroup $S$ of $G$ such that $|s|=d_{i}$, let $D_{i}(S)$ be the set of o,l unfeithfil complexes of length , where $l \leq b_{i}$, end uncieryying subgroup $s$. Then ve have that

$$
D_{i}(S)=x_{b_{i}}(S) \cup\left[\left(S_{b_{j}}\left(T_{j y}\right) \mid i+I \leq j \leq t \text { and } I \leq y \leq x_{j}\right\}\right]
$$

where for each $j,(i+l \leqslant j \leq t)$, the set $\left\{T_{j l}, T_{j 2}, \cdots, T_{j x_{j}}\right\}$ is the set of a.1. subgrovis or order d which contain $s$. Nioneover, this representa,tion of $D_{i}(S)$ as a set union is peirrrise disjoint.

Proof. Let $D_{1}$ denote the set union on the right in (3.3). By Lemma 3.1. part (b) we know that the set union $D_{1}$ is pairwise disjoint. Suppose that $I \in D_{1}$. Then either $H \in X_{b_{1}}(S)$ or $H \in X_{b_{j}}\left(T_{j y}\right)$ for exactiy one $j$ and one $y$ such that $i+I \leq j \leq t, I \leq y \leq x_{j}$. If $H \in \mathrm{X}_{\mathrm{b}_{i}}(S)$, then clearly $\mathrm{H} \in \mathrm{D}_{i}(S)$ by definition of the set $D_{i}(S)$. If $H \in X_{b_{j}}\left(T_{j y}\right)$, then $H$ is a disjoint union of cosets belonging to $T_{j y}$. Since $T_{j y}$ contains $S$, it follows that $H$ is a disjoint wion of cosets belonging to $\mathrm{S}$ so that $\mathrm{S}$ underlies $\mathrm{H}$. Hence $H \in D_{i}(S)$. On the other hand suppose that $H \in D_{i}(S)$. Then by Corollary 2.3 . $H$ has a unique length $l$ and by Proposition 2.3 . we must have that $l=b_{j}$ 
for exactly one $j(i \leq j \leq t)$. Suppose that $T$ is the induced subgroup for H. By Theorem 2.2. it follows, since $S$ underlies $H$, that $S \subseteq T$ and by Lemma 3.1. part (a) that $|T|=d_{j}$. Hence $H \in X_{b_{j}}(T) \subseteq D_{1}$, that is, $H \in D_{1}$. Thus $D_{i}(S)=D_{I}$ and this completes the proof.

Lemma 3.4. Let $T$ be a subgroup of $G$ of order $d_{j}$ and let $i$ be any number such that $1 \leq i<j \leq t$. Each unfaithful complex $H \in X_{b_{j}}$ (T) gives rise to exactly $\psi_{G}\left(d_{i}, T\right)=\left|R\left(K_{d_{1}}, T\right)\right|$ representations of $H$ as disjoint unions of cosets belonging to some subgroup of order $d_{i}$. Hence the total number of distinct representations of the members of the set $\mathrm{x}_{\mathrm{b}_{j}}\left(\mathrm{~K}_{\mathrm{d}_{j}}\right)$ as disjoint set unions of cosets belonging. to subgroups $s$ of order $d_{i}$ is given by $\sum_{y=1}^{\psi_{G}\left(d_{j}\right)} \psi_{G}\left(d_{i}, T_{j y}\right)\left|x_{b_{j}}\left(T_{j y}\right)\right|$, where $\left\{T_{j y} \mid I \leq y \leq \psi_{G}\left(d_{j}\right)\right\}$ is the set of all subgroups of $G$ of order $d_{j}$.

Proof. By Theorem 2.2. we know that $\mathrm{H} \in \mathrm{X}_{\mathrm{b}_{\mathrm{j}}}(\mathrm{T})$ has a nonzero underlying subgroup $S$ if and only if $S \subseteq S_{H}$ where $S_{H}$ is the subgroup induced by $H$. By Lemma 3.1. we have, since $|T|=d_{j}$, that $S_{H}=T$. Hence $H$ has as many distinct representations as a disjoint union of cosets belonging to some subgroup $\mathrm{S}$ of order $d_{i}$ as there are subgroups $\mathrm{S} \subseteq \mathrm{T}$ of order $d_{i}$. Thus $\mathrm{H}$ gives rise to $\psi_{G}\left(d_{i}, T\right)$ representations with underlying subgroup of order $d_{i}$. By Lemma 3.1 , part $(b)$, we know that $x_{b_{j}}\left(K_{d_{j}}\right)=U\left\{x_{b_{j}}\left(T_{j y}\right) \mid I \leq y \leq \psi_{G}\left(d_{j}\right)\right\}$ and that this union is pairwise disjoint. It now follows immediately that the total number of representations of the members of the set $x_{b_{j}}\left(K_{d_{j}}\right)$ in terms of underlying subgroups of order $a_{i}$ is given by ${ }_{y=1}^{\psi_{G}\left(d_{j}\right)} \psi_{G}\left(d_{i}, T_{j y}\right)\left|x_{b_{j}}\left(T_{j y}\right)\right|:$ This completes the proof. 
We now in the next result derive a recursive formula giving the cardinality of the set of complexes of order $v$ and length $\ell$ which have a fixed subgroup $S$ as their induced subgroup.

Theorem 3.1. For each $i,(1 \leq i \leq t)$, the cardinality of the set of all unfaithful complexes of order $\nu$ and length $b_{i}$ which have an induced subgroup $s$ of order $d_{i}$, that is, the cardinality of the set $x_{b_{1}}(s)$ where $S$ is a subgroup of order $d_{i}$, is given by the following recursive formula: For $i=t$ so that $d_{t}=d, b_{t}=b$ and $r_{t}=r$ we have

$$
\left|x_{b}(s)\right|=c_{b}^{r}
$$

and for $1 \leq i \leq t-1$ we have

$$
\left|x_{b_{1}}(s)\right|=c_{b_{1}}^{r_{i}}-\sum_{j=i+1}^{t} \sum_{y=1}^{x_{j}}\left|x_{b_{j}}\left(T_{j y}\right)\right|
$$

where for each $j,(i+1 \leq j \leq t),\left\{T_{j_{1}}, \cdots, T_{j x_{j}}\right\}$ is the set of all subgroups of $G$ of order $d_{j}$ which contain $S$.

Proof. Note that by Lemma 3.1., since $|s|=d_{i}$, that $x_{b_{1}}$ (s) is the set of all unfaithful complexes of order $v$ and length $b_{i}$ with induced subgroup S. Suppose that $i=t$. Then $S$ has order $d_{t}=d$ and index $r_{t}=r$. Hence there are $c_{b}^{r}$ possible unions of $b$ distinct cosets from the cosets of $\mathrm{S}$. Select one such union of $b$ cosets, say $H=\left(s+a_{1}\right) \cup \cdots U\left(s+a_{b}\right)$. We claim that $H$ has length $b$. Let $S_{H}$ be the subgroup induced by $H$. Then by Theorem 2.2. we have that $S \subseteq S_{H}$ and thus $d=|S| \leq\left|S_{H}\right|$. But by Proposition 2.2. we have that $\left|S_{H}\right|$ divides $d$. It follows then that $S=S_{H}$ and $v=k d=\left(k m_{t}\right) d_{t}=b_{t}\left|s_{H}\right|=b\left|s_{H}\right|$. Hence by Theorem 2.3. we have that $H$ has length $b$, that is, $H \in X_{b}(s)$. Thus we have that $\left|X_{b}(s)\right|=c_{b}^{r}$ and (3.4) 
is established. Let $I \leq i \leq t-1$ and let $D_{i}(s)$ be the set introduced in Lemma 3.3. Then $S$ has order $d_{i}$ and index $r_{i}$ so that the cardinality of the set of all unfaithful complexes of order $v$ and underlying subgroup $S$ is $C_{b_{1}}^{r_{i}}$, that is, $\left|D_{i}(S)\right|=C_{b_{i}}^{r_{i}}$. Since the representation for $D_{i}(S)$ given in (3.3) is a pairwise disjoint union, it follows immediately using (3.3) that $\left|D_{i}(s)\right|=c_{b_{i}}^{r_{i}}=\left|x_{b_{i}}(s)\right|+\sum_{j=i+1}^{t} \sum_{y=1}^{x_{j}}\left|x_{b_{j}}\left(T_{j y}\right)\right|$ and (3.5) follows by rearrangement. This completes the proof.

Theorem 3.2. For each i, (1sist), the cardinality of the set of all unfaithful complexes of order $v$ and length $b_{i}$ which have an induced subgroup of order $d_{i}$, that is, the cardjnality of the set $x_{b_{1}}\left(K_{d_{1}}\right)$ is given by the following recursive foxnula: For $i=t$, so that $d_{t}=d, b_{t}=b$ and $r_{t}=r$ :

$$
\left|x_{b}\left(K_{d}\right)\right|=\psi_{G}(d) c_{b}^{r}
$$

and for $i$ such that $1 \leq i \leq t-1$ :

$$
\left|x_{b_{1}}\left(K_{d_{1}}\right)\right|=\psi_{G}\left(a_{i}\right) c_{b_{1}}^{r_{i}}-\sum_{j=i+1}^{t} \sum_{y=1}^{\psi_{G}\left(d_{j}\right)} \psi_{G}\left(d_{i}, T_{j y}\right)\left|x_{b_{j}}\left(T_{j y}\right)\right|
$$

where $\left\{T_{j y} \mid I \leq y \leq \psi_{G}\left(d_{j}\right)\right\}$ is the set of all subgroups of $G$ of order $d_{j}$ and $\left|x_{b_{j}}\left(T_{j y}\right)\right|(i+1 \leq j \leq t)$ is given by $(3.4)$ and $(3.5)$.

Proof. By Lemma 3.1., part (b), we have that $X_{b}\left(K_{d}\right)=U\left\{X_{b}(s) \mid s \in K_{d}\right\}$ and that this union is pairwise disjoint. Hence $\left|x_{b}\left(K_{d}\right)\right|=\Sigma\left|x_{b}(S)\right|$ where we sum over all $S \in K_{d}$. By $(3.4),\left|X_{b}(S)\right|=c_{b}^{r}$ for any $S \in K_{d}$. It follows immediately that $\left|x_{b}\left(K_{d}\right)\right|=\psi_{G}(d) c_{b}^{r}$ and formula (3.6) is established. Now choose any $i$ such that $I \leq i \leq t-1$. There are $\psi_{G}\left(d_{i}\right)$ subgroups of $G$ 
of order $d_{i}$ and each such subgroup $S$ produces $c_{h_{i}}{ }_{i}$ unfaithful complexes whose underlying subroup is 5 . Hence the total numer of unaithful complexes of order $v$ with an arcentying suberen of order $a_{i}$ is ${ }_{G}\left(d_{i}\right) a_{b_{1}}^{r}$. Now by

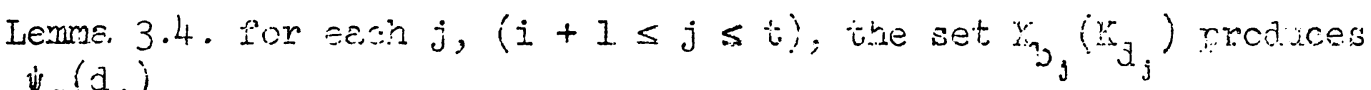
$\left(\mathrm{W}_{\mathrm{s}}\left(\mathrm{d}_{j}\right)\right.$

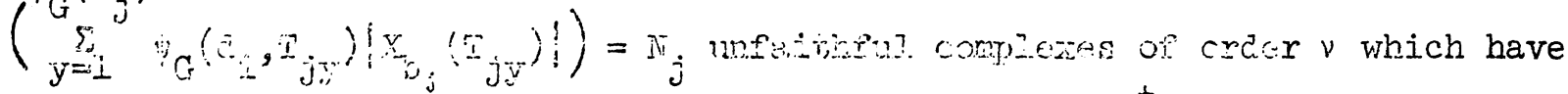

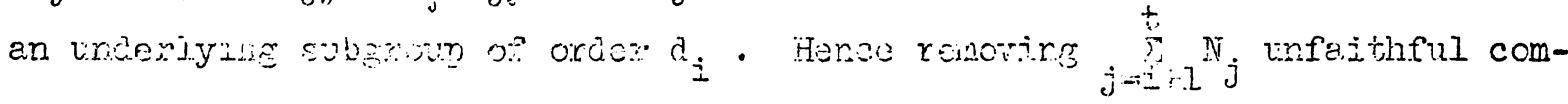
plexes of onsex y wich hate an unde:lying suremoup of order $d_{i}$ from amongst

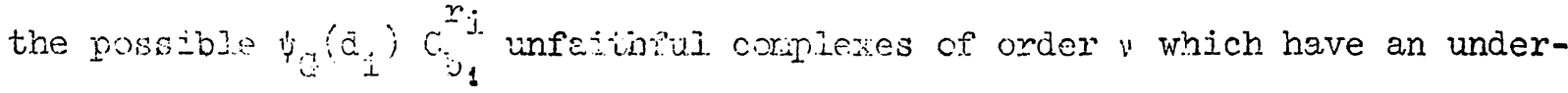
lying stibgroup of oreier $d_{i}$, which leaves us with only those unfaithful

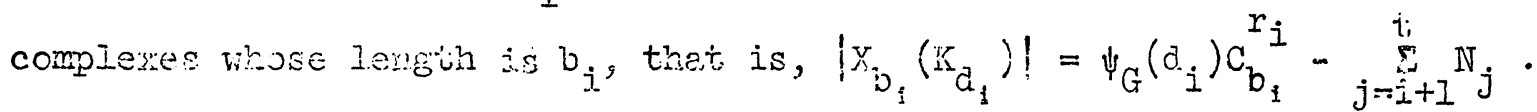
Hence fompula $(3.7)$ is errablisted and this completes the proof.

$$
\text { For each } i,(I \leq i \leq t) \text {, let } c_{i}=\left|x_{b_{1}}\left(K_{d_{1}}\right) / \tau\right| \text {, that is, let } c_{i} \text { be }
$$
the number of eqpivalence classes into which the set $\mathrm{x}_{\mathrm{b}_{1}}\left(\mathrm{~K}_{\mathrm{d}_{1}}\right)$ is decomposed under the relatiois $\tau$. We let $c_{i}=0$ in case $x_{b_{1}}\left(K_{d_{1}}\right)=\phi$.

Theorem 3.3. For each i, $(I \leq i \leq t)$, the number of equivalence classes $c_{i}$ into winich the set. $x_{b_{1}}\left(K_{d_{1}}\right)$ is decomposed under $\tau$ is given by $c_{i}$ $=\frac{1}{r_{i}}\left|\mathrm{X}_{\mathrm{b}_{1}}\left(\mathrm{~K}_{\mathrm{a}_{1}}\right)\right| \cdot$ Isores the least number of unfaithful complexes of order $v$, length $b_{i}$ and jngreed subgroul of order $d_{i}$, which will generate the set $\mathrm{x}_{\mathrm{b}_{1}}\left(\mathrm{~K}_{\mathrm{d}_{1}}\right)$ under the action of $\tilde{\alpha}\left(\mathrm{G}\right.$; is given by $\frac{1}{\mathrm{r}_{1}}\left|\mathrm{~K}_{\mathrm{b}_{1}}\left(\mathrm{~K}_{\mathrm{d}_{1}}\right)\right|$.

Proof. Let $\left\{S_{i y} \mid I \leq y \leq \psi_{G}\left(d_{j}\right)\right\}$ be the set of all subgroups of order $d_{i}$. By Leruma 3.I., part (b) aind part (c), we heve cleanly that $x_{b_{1}}\left(K_{d_{1}}\right) / \tau=U\left\{x_{b_{i}}\left(s_{i y}\right)_{/ \tau}: I \leq y \leq \psi_{G}\left(d_{i}\right)\right\}$. Hence $c_{i}=\left|x_{b_{1}}\left(K_{d_{1}}\right) / \tau\right|$ $\dot{\psi}_{G}\left(z_{\dot{i}}\right)$

$=\sum_{y=i}\left|\mathrm{x}_{\mathrm{b}_{1}}\left(\mathrm{~s}_{i-\mathrm{y}}\right) / \tau\right|$. Fur each subgroup $\mathrm{S}$ of order $\mathrm{d}_{i}$ let $x_{S}=\left|\mathrm{x}_{\mathrm{b}_{1}}(\mathrm{~s}) / \tau\right|$. 
Pick any $H \in \mathrm{X}_{\mathrm{b}_{1}}(\mathrm{~S})$; then as remarked in (3.2) we have that $[\mathrm{H}]_{\tau}=\tilde{\Omega}(\mathrm{G}) * \mathrm{H}$ and by Lemma 3.1., $[\mathrm{H}]_{\tau} \subseteq \mathrm{X}_{\mathrm{b}_{1}}(\mathrm{~S})$ and $\mathrm{S}$ is the subgroup induced by $\mathrm{H}$, that is, $S=S_{H}$. Hence by Theorem 2.5., $\left|[\mathrm{H}]_{\tau}\right|=|G / S|=r_{i}$. It follows that $\left|x_{b_{1}}(s)\right|=x_{S} r_{i}$, so that $x_{S}=\left|x_{b_{1}}(s) / \tau\right|=\frac{1}{r_{1}}\left|x_{b_{1}}(s)\right|$. Hence $c_{i}=\sum_{y=1}^{\psi_{G}}\left(d_{i}\right) \frac{1}{r_{i}}\left|x_{b_{1}}\left(s_{i y}\right)\right|=\frac{1}{r_{i}}\left|x_{b_{1}}\left(K_{d_{1}}\right)\right|$, for by Lemma 3.1. $x_{b_{1}}\left(K_{d_{1}}\right)$ $=U\left\{\mathrm{x}_{\mathrm{b}_{1}}\left(\mathrm{~s}_{\mathrm{iy}}\right) \mid \mathrm{I} \leq \mathrm{y} \leq \psi_{\mathrm{G}}\left(\mathrm{d}_{\mathrm{i}}\right)\right\}$, the union being pairwise disjoint. This completes the proof.

Corollary 3.1. The cardinality of the quotient set $U_{\nu} / \tau$ is given by $\sum_{i=1}^{t} \frac{1}{r_{i}}\left|x_{b_{1}}\left(k_{d_{1}}\right)\right|$ where for each $i,(1 \leq i \leq t)$, the number $\left|x_{b_{1}}\left(k_{d_{1}}\right)\right|$ is given by the recursive formulae (3.6) and (3.7).

Proof. By Lemma 3.2. and Lemma 3.1., part (c), it follows that $\left|U_{\nu / \tau}\right|$ $=\sum_{i=1}^{t}\left|x_{b_{i}}\left(K_{d_{1}}\right) / \tau\right|$. Using Theorem 3.3. we have that $\left|U_{v} / \tau\right|=\sum_{i=1}^{t} \frac{1}{r_{i}}\left|x_{b_{1}}\left(K_{d_{i}}\right)\right|$ and this completes the proof.

Theorem 3.4. The cardinality $f$ of the quotient set $F_{\nu} / \tau$ is given by

$$
f=\frac{1}{n}\left[c_{v}^{n}-\sum_{i=1}^{t} c_{i} r_{i}=\frac{1}{n}\left[c_{v}^{n}-\left|u_{v}\right|\right]\right.
$$

Thus, in particular, the set consisting of faithful complexes of order $v$ one from each equivalence class in $F_{\nu / \tau}$ which generate all of $F_{\nu}$ under the action of the group $\tilde{\Omega}(G)$ has cardinality $f$, given by formula (3.8).

Proof. By Theorem 2.5. if $H \in F_{\nu}$ then $\left|[H]_{\tau}\right|=|\tilde{\Omega}(G) * H|=n$. Hence $\left|F_{v}\right|=$ fn where $f=\left|F_{v} / \tau\right|$. Further if $H \in X_{b_{1}}\left(K_{d_{1}}\right)$, then by Theorem 2.5. $\left|[H]_{\tau}\right|=\left|G / S_{H}\right|=r_{i}$ for the subgroup induced by $H$ has order $d_{i}$. Since 
$c_{i}=\left|x_{b_{1}}\left(K_{d_{1}}\right)\right|$ and each equivalence class in $x_{b_{1}}\left(K_{d_{1}}\right)$ has cardinality $r_{i}$, it follows that $\left|\mathrm{x}_{b_{1}}\left(\mathrm{~K}_{\mathrm{d}_{1}}\right)\right|=c_{i} r_{i}$. Then by Lemma 3.2. we have that $\left|U_{v}\right|$ $=\sum_{i=1}^{t}\left|x_{b_{1}}\left(K_{d_{1}}\right)\right|=\sum_{i=1}^{t} c_{i} r_{i}$. Since $\left|c_{v}\right|=c_{v}^{n}$ and $c_{v}=F_{v} \cup U_{v}$ is a disjoint union, $\left|c_{v}\right|=c_{v}^{n}=\left|F_{v}\right|+\left|U_{v}\right|=f n+\sum_{i=1}^{t} c_{i} r_{i}$, and formula (3.8) is obtained by rearrangement. This completes the proof.

Corollary 3.2. The cardinality $c$ of the quotient set $C_{\nu / \tau}$ is given by

$$
\left.c=\frac{1}{n} c_{\nu}^{n}-\sum_{i=1}^{t} c_{i} r_{i}\right\rceil+\sum_{i=1}^{t} c_{i}
$$

where for each $i,(1 \leq i \leq t), c_{i}=\frac{1}{r_{1}}\left|x_{b_{1}}\left(K_{d_{1}}\right)\right|$ and the number $\left|x_{b_{1}}\left(K_{d_{1}}\right)\right|$ is given by the recursive formulae (3.6) and (3.7). Thus, in particular, the set consisting of complexes of order $v$, one from each equivalence class in $C_{\nu} / \tau$ which generates all of $C_{\nu}$ under the action of the group $\tilde{\Omega}(G)$, has cardinality $c$ given by (3.9).

Proof. Note that since $C_{\nu}=F_{\nu} \cup U_{\nu}$, a disjoint union, we have immediately that $\left|C_{\nu} / \tau\right|=\left|F_{\nu} / \tau\right|+\left|U_{\nu} / \tau\right|$, using Lemma 3.1., part (c). Now using Theorem 3.4. and Corollary 3.1. we immediately obtain formula (3.9). This completes the proof.

In the next result we obtain a formula which counts the number of complexes of order $v$ which contain the zero element of $G$.

Theorem 3.5. (i) The number of unfaithful complexes of order $v(v \geq 2)$ in $G$ which contain the zero element of $G$ is given by $\sum_{i=1}^{t} c_{i} b_{i}$. (ii) The number of faithful complexes of order $\vee(\nu \geq 2)$ in $G$ which contain the zero element of $G$ is given by $f \nu$ where $f=\left|F_{\nu} / \tau\right|$. Hence the total number of 
complexes of order $\nu(\nu \geq 2)$ in G which contain the zero of $G$ is given by $\sum_{i=1}^{t} c_{i} b_{i}+f v$ and in particular $\sum_{i=1}^{t} c_{i} b_{i}+f v=c_{v-1}^{n-1}$.

Proof. (i) Pick any unfaithful complex $\mathrm{H}$ in $\mathrm{U}_{v}$ containing the zero element of $G$. Then by Lemma 3.2., $H \in X_{b_{1}}\left(K_{d_{1}}\right)$ for exactly one $i(1 \leq i \leq t)$. Thus there exists a group $S$ of order $d_{i}$ which is the group induced by $H$ such that $\mathrm{H}=\mathrm{S} \cup\left(\mathrm{s}+\mathrm{a}_{1}\right) \cup \cdots \cup\left(\mathrm{s}+\mathrm{a}_{\mathrm{b}_{1}-1}\right)$, a disjoint union: Clearly the sets $H, H+\left(-a_{1}\right), H+\left(-a_{2}\right), \cdots, H+\left(-a_{b_{1}-1}\right)$ are all distinct; otherwise, in case $H+\left(-a_{i}\right)=H+\left(-a_{j}\right)$, it follows that $a_{j}-a_{j} \in S=S_{H}$ and thus $s+a_{i}=s+a_{j}$, a contradiction to the choice of the $a_{i}$. Further these sets $H, H+\left(-a_{1}\right), \cdots, H+\left(-a_{b_{1}-1}\right)$ all belong to $[H]_{\tau}$. Hence there are exactly $b_{i}$ sets in each equivalence class $[H]_{T}$ which contain the zero of $G$. Now by definition $c_{i}=\left|x_{b_{1}}\left(K_{d_{1}}\right) / \tau\right|$ and hence it follows that there are exactly $c_{i} b_{i}$ unfaithful complexes in $\mathrm{X}_{\mathrm{b}_{1}}\left(\mathrm{~K}_{\mathrm{d}_{1}}\right)$ which contain the zero of $\mathrm{G}$. Using Lemma 3.2. we immediately obtain that there are $\sum_{i=1}^{t} c_{i} b_{i}$ unfaithful complexes of order $\nu$ containing the zero element of $G$. (ii) Pick any $H$ in $F_{v}$, and suppose $H=\left\{a_{1}, a_{2}, \cdots, a_{v}\right\}$. Then clearly the sets $H+\left(-a_{l}\right)$, $H+\left(-a_{2}\right), \cdots, H+\left(-a_{\nu}\right)$ are all distinct, since $H$ is faithful, and each contains the zero element of $G$. Hence in each equivalence class $[H]_{\tau}$ with $\mathrm{H}$ in $\mathrm{F}_{v}$ there are precisely $\nu$ faithful complexes of order $v$ each containing the zero of $G$. Since $\left|F_{\nu / \tau}\right|=f$, it follows that there are precisely fv faithful complexes of order $v$ in $G$ containing the zero element of $G$, and (ii) is established. Finally consider the set $G^{\prime}=G-\{0\}$ where 0 is the zero element of $G$. There are exactly $C_{\nu-I}^{n-I}$ sets of order $\nu-1$ in $G^{\prime}$. If $\nu \geq 2$ then each of these sets is nonempty and lacks the zero of $G$. Adding the zero element of $G$ to each of these sets gives $c_{\nu-1}^{n-1}$ sets of order $v$ in $G$ 
containing the zero element of $G$. It follows that $\sum_{i=1}^{t} c_{i} b_{i}+f v=c_{v-I}^{n-I}$.

Corollary 3.3. If the g. $\underline{\text { c. }}$. $\cdot(v, n)=1$ and $v \geq 2$ the $U_{v}=\varnothing$ and the cardinality $f$ of the quotient set $F_{v / \tau}$ is $f=\frac{1}{v} c_{v-1}^{n-1}$. If $v=1$, then $f=1$. In particular if the $\mathrm{g} \cdot \underline{\mathrm{c}} \cdot \underline{\mathrm{d}} \cdot(v, \mathrm{n})=1$, then $v$ divides $\mathrm{C}_{v-1}^{\mathrm{n}-1}$ and $\mathrm{n}$ divides $c_{v}^{n}$

Proof. By Proposition 2.4. we know that if the g.c.d. $(v, n)=1$ that $U_{v}=\varnothing$. Hence if $v \geq 2$ the formula given in Theorem 3.5. reduces to fv $=c_{v-1}^{n-1}$ and thus $f=\frac{1}{v} c_{v-1}^{n-1}$. If $v=1$ it is clear that $f=1$. Also in the case that the g.c. a. $(v, n)=1$ formula (3.8) reduces to $f=\frac{1}{n} c_{v}^{n}$ so that $n$ divides $C_{v}^{n}$. This completes the proof.

Suppose that $K$ is an abelian group such that $K$ is a direct product, $K=\underset{i=1}{\alpha} C_{i}$, where for each $i(I \leq i \leq \alpha) c_{i}$ is a cyclic group and the order of each $C_{i}$ is a fixed prime $p$. Then $|K|=p^{\alpha}$. The following formula giving the number of subgroups of $K$ of order $p^{\beta}$ is well known, for example, see Carmichael [1956]:

$$
\psi_{K}\left(p^{\beta}\right)=\frac{\left(p^{\alpha}-1\right)\left(p^{\alpha}-p\right) \cdots\left(p^{\alpha}-p^{\beta-1}\right)}{\left(p^{\beta}-1\right)\left(p^{\beta}-p\right) \cdots\left(p^{\beta}-p^{\beta-1}\right)}
$$

In the next result $G_{0}$ will stand for either a cyclic group of order $n$ or an abelian group of order $n$ of the form $G_{0}=\left(c_{p_{1}} \times \cdots \times c_{p_{1}}\right) \times\left(c_{p_{?}} \times \cdots \times c_{p_{2}}\right)$ $* \cdots \times\left(c_{p_{m}} \times \cdots \times{ }_{p_{m}}\right)$ where for each $i,(1 \leq i \leq m), c_{p_{1}}$ is a cyclic group of order $p_{i}, p_{1}, \cdots, p_{m}$ are distinct primes and the group $c_{p_{1}}$ occurs $\alpha_{i}$ times. Thus in this case $n=p_{1}^{\alpha_{1}} p_{2}^{\alpha_{3}} \ldots p_{m}^{\alpha_{m}}$. 
Let $G_{i}=C_{p_{1}} \times C_{p_{1}} \times \cdots \times C_{p_{1}}$ so that $G_{0}=G_{1} \times G_{2} \times \cdots * G_{m}$. Then it is clear that the number of subgroups of $G_{0}$ of order $s=p_{1}^{\beta_{1}} p_{2}^{\beta_{1}} \cdots p_{m}^{\beta_{m}}$ is the number $\psi_{G_{0}}(s)=\psi_{G_{1}}\left(p_{1}^{\beta_{1}}\right) \psi_{G_{m}}\left(p_{2}^{\beta_{2}}\right) \cdots \psi_{G_{m}}\left(p_{m}^{\beta_{m}}\right)$ where $\psi_{G_{1}}\left(p_{i}^{\beta_{1}}\right)$ is given by the formula (3.10).

Note further that any two subgroups of $G_{0}$ of the same order are isomorphic. For each $i,(1 \leq i \leq t)$, let $s_{i}$ be a subgroup of $G_{0}$ of order $d_{i}$ and for any $j$ such that $I \leq i<j \leq t$ let $\psi_{G_{0}}\left(d_{i}, S_{j}\right)=a_{i j}$. Then by the remark just made if $T$ is any subgroup of $G_{0}$ of order $d_{j}$ we have immediately that $\psi_{G_{0}}\left(d_{i}, s_{j}\right)=\psi_{G_{0}}\left(d_{i}, T\right)=a_{i j}$ for each $i, j(I \leq i<j \leq t)$. In the next result we show that the formulae (3.6) and (3.7) reduce to formulae involving the numbers $a_{i j}$ in the case the group $G$ has the form of $G_{0}$.

Theorem 3.6. For each i $(1 \leq i \leq t)$ the cerainality of the set of all unfajthful complexes of $G_{0}$ of order $\nu$ and lengith $b_{i}$ which have an induced subgroup of order $d_{i}$ is given by the following formula: For $i=t$ so that $a_{t}=d, b_{t}=b$ and $r_{t}=r$,

(3.11) $\quad\left|x_{b}\left(K_{d}\right)\right|=\psi_{G_{0}}(d) c_{b}^{r}$

and for $I \leq i \leq t-1$

(3.12) $\left|x_{b_{1}}\left(k_{d_{i}}\right)\right|=\psi_{G_{0}}\left(a_{i}\right) c_{b_{1}}^{r_{i}}-\sum_{j=i+1}^{t} a_{i j}\left|x_{b_{j}}\left(K_{d_{j}}\right)\right|$ and $a_{i j}=\psi_{G_{0}}\left(d_{i}, s_{j}\right)$.

Proof. Since formula (3.6) holds for an arbitrary group G it holds for the case $G=G_{0}$ as well. Hence (3.11) follows from (3.6) with $G$ replaced by $G_{0}$. Next let $i$ be any number such that $1 \leq i \leq t-1$. We have 
seen above that if $T$ is any subgroup of order $d_{j},(i+1 \leq j \leq t)$, then $\Psi_{G_{0}}\left(d_{i}, s_{j}\right)=\Psi_{G_{0}}\left(d_{i}, T\right)=a_{i j}$. Hence in this case formula (3.7) reduces to $\left|\mathrm{x}_{\mathrm{b}_{1}}\left(\mathrm{~K}_{\mathrm{d}_{1}}\right)\right|=\psi_{\mathrm{G}_{0}}\left(\mathrm{~d}_{i}\right) \mathrm{c}_{\mathrm{b}_{1}}^{r_{1}}-\sum_{j=i+1}^{t} a_{i j}\left[\sum_{y=1}^{\mathrm{G}_{0}}{ }^{\left(\mathrm{d}_{j}\right)}\left|\mathrm{x}_{\mathrm{b}_{j}}\left(\mathrm{~T}_{j y}\right)\right|\right]$. Since by Lemma 3.I. $\mathrm{X}_{\mathrm{b}_{j}}\left(\mathrm{~K}_{\mathrm{d}_{j}}\right)=U\left\{\mathrm{X}_{\mathrm{b}_{j}}\left(\mathrm{~T}_{j \mathrm{y}}\right) \mid I \leq \mathrm{y} \leq \Psi_{\mathrm{G}_{0}}\left(\mathrm{~d}_{j}\right)\right\}$, a disjoint union, we have immediately that $\left|x_{b_{j}}\left(K_{d_{j}}\right)\right|=\Sigma\left|x_{b_{j}}\left(T_{j y}\right)\right|$ and formula (3.12) is established. This completes the proof.

We illustrate some of the ideas developed thus far in this section with the following example:

Example 3.1. Let $G=\underset{i=1}{7} C_{i}$ be the direct product of seven cyclic groups $C_{i}$ each of order 2. Then $|G|=n=128$. Let $v=8$ so that the g. c. $d \cdot(v, n)=d=8$. Hence the set of dirisors of d distinct from 1 are $D=\{2,4,8\}$. Let $d_{1}=2, d_{2}=4, d_{3}=8$, then $b_{1}=4, b_{2}=2, b_{3}=1$ so that the set $L=\{1,2,4\}$, and $r_{1}=64, r_{2}=32$, and $r_{3}=16$. Let $S_{1}$ and $\mathrm{S}_{2}$ be subgroups of $\mathrm{G}$ of orders 4 and 8 respectively. Using formula (3.10) we now have that $(i) \psi_{G}(2)=127, \psi_{G}(4)=2667, \psi_{G}(8)=11,811$ and that (ii) $\psi_{G}\left(2, S_{1}\right)=3, \psi_{G}\left(4, S_{2}\right)=7, \psi_{G}\left(2, S_{2}\right)=7$. Now using Theorem 3.6. the number of unfaithful complexes of length $I$ is $\left|\mathrm{X}_{1}\left(\mathrm{~K}_{\mathrm{d}}\right)\right|=\left|\mathrm{X}_{1}\left(\mathrm{~K}_{8}\right)\right|=\psi_{\mathrm{G}}(8) \mathrm{C}_{1}^{16}$. The number of unfaithful complexes of length 2 is $\left|\mathrm{x}_{2}\left(\mathrm{~K}_{4}\right)\right|=\left(\psi_{G}(4)\right) \mathrm{C}_{2}^{32}$ - $\left[\psi_{G}(8) C_{1}^{16} \psi_{G}\left(4, S_{2}\right)=0\right.$. The number of unfaithful complexes of length 4 is $\left|X_{4}\left(K_{2}\right)\right|=\psi_{G}(2) c_{4}^{64}-\left|x_{2}\left(K_{4}\right)\right|_{G}\left(2, s_{1}\right)-\left|x_{1}\left(K_{8}\right)\right| \psi_{G}\left(2, s_{2}\right)=79,369,920$. Hence using Lemma 3.2. the total number of unfaithful complexes of order 8 , that is, $\left|\mathrm{U}_{8}\right|=\left|\mathrm{x}_{1}\left(\mathrm{~K}_{8}\right)\right|+\left|\mathrm{x}_{2}\left(\mathrm{~K}_{4}\right)\right|+\left|\mathrm{x}_{4}\left(\mathrm{~K}_{2}\right)\right|=79,558,896$. Using formula (3.8) of Theorem 3.4. the cardinality $f$ of the quotient set $F_{\nu / \tau}$ is $f=\frac{1}{128}\left[c_{8}^{128}-\left|U_{8}\right|\right]=11,168,930,418$. Using Corollary 3.1. the cardi- 
nality $u$ of the quotient set $U_{8 / \tau}$ is $u=\frac{\left|x_{1}\left(K_{8}\right)\right|}{16}+\frac{\left|x_{2}\left(K_{4}\right)\right|}{32}+\frac{\left|x_{4}\left(K_{2}\right)\right|}{64}$ $=1,251,966$. Finally using formula (3.9) of Corollary 3.2. the cardinality $c$ of the quotient set $c_{8 / \tau}$ is $c=f+u=11,170,182,384$. Hence if one selects $c$ sets one from each equivalence class in $C_{8 / \tau}$ the resulting set is the set of least cardinality such that the action of $\tilde{\Omega}(G)$ on it generates all of $\mathrm{C}_{8}$

Paik and Federer [1970] proposed the name "generator" for both faithful and unfaithful complexes of a group and this term was subsequently used by Raktoe and Federer [1971]. We have departed in this paper from this terminology because the term generator has a distinct meaning in group theory. Raktoe and Federer [1971] gave the following formula relating the cardinalities of $\mathrm{C}_{V}, \mathrm{~F}_{V}$, and $\mathrm{U}_{V}$ :

$$
\text { (3.13) } \quad\left(\psi_{G}(v)\right) a+f\left(s^{t}\right)=c_{v}^{s^{t}}
$$

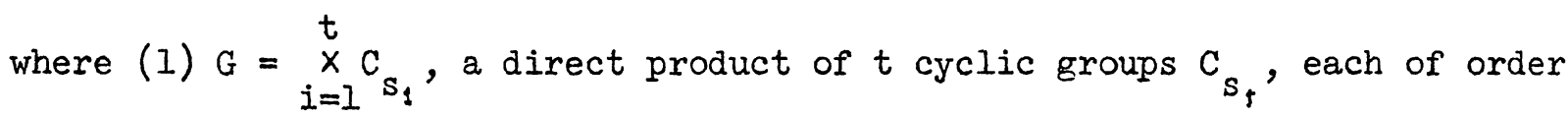
$s_{i}=s$ and $s=s_{i}=p^{\alpha}$ for each $i, p$ is a fixed prime, and $\alpha \geq 1$ is a fixed number, (2) $v=t(s-1)+1,(3) a=$ index of a subgroup of order $v$, and (4) $f=$ cardinality of the quotient set $F_{V / \tau}$.

The theory developed thus far shows that the R-F formula is not true in general, since the formula assumes that all unfaithful complexes of order $v$ are of length one, that is, it assumes that all unfaithful complexes of order $v$ are subgroups of $G$ or cosets belonging to a subgroup of $G$ of order $\nu$. Since unfaithful complexes of length other than one do exist in general, it is clear that the number $f$ in formula (3.13) merely provides an 
upper bound to the cardinality of $F_{V / \tau}$. A comparison of formula (3.13) with formula (3.8) which gives the cardinality of $F_{\nu / \tau}$ illustrates that those unfaithful complexes of order $v$ and length other than one have not been taken into account in formula (3.13). We now present a counter example to formula (3.13) by exhibiting an unfaithful complex of length other than one in a particular case.

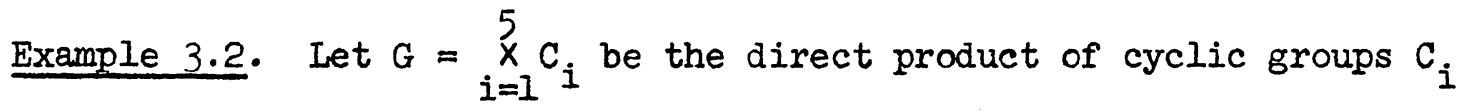
where for each $i, c_{i}=\{0,1\}$ under addition mod. 2. Hence in this case $s^{t}=2^{5}$ and $v=6$. Since there are no subgroups of order $\operatorname{six}$ in $G$, we have that $\psi_{G}(6)=0$ so that according to formula $(3.13) f\left(2^{5}\right)=c_{6}^{2^{5}}$ which implies that $2^{5}=32$ divides $\mathrm{C}_{6}^{32}$. This, however, is impossible. Further there are unfaithfui complexes of order six. For example, take $H\{(0,0,0,0,0),(\lambda, 0,0,0,0)$, $(0,1,0,0,0),(1,1,1,1,1),(0,1,1,1,1),(1,1,0,0,0)\}$. Then 1 ia un itfoftinit oomplex of Iength tinree whosic unique indued aubgroup is $\{(0,0,0,0,0),(1,0,0,0,0)\}$.

We now give necessary and sufficient conditions under which the formula (3.13) holds where we remove the restriction placed on $v$ as given in (2) and allow $v$ to be any given number such that $I \leq v \leq s^{t}$. The following lemmas will be useful.

Lemma 3.5. Suppose that $\mathrm{C}_{\mathrm{s}}=\{0,1,2, \cdots, \mathrm{s}-1\}$ is a cyclic group under addition mod. $s$ and let $s=p^{\alpha}$ where $p$ is a prime and $\alpha$ is any number such that $\alpha>1$. Then for each $i, 1<i<\alpha$, there exists an unfaithful complex of order $\mathrm{p}^{i}$ and length $\mathrm{p}$ in $\mathrm{C}_{\mathrm{s}}$.

Proof. Let $K$ be the cyclic subgroup of $C_{S}$ of order $p^{i-1}$ generated by $p^{\alpha-i+1}$, that is, $K=\left\{m p^{\alpha-i+1} / 0<m \leq p^{i-1}\right\}$. Since $i>1$, we have that 
$|K|=p^{i-1} \neq I$ so that $K$ is a nonzero subgroup of $C_{S}$. Since $\alpha>1$, the set of positive numbers $S=\{1,2, \cdots, p-1\}$ less than $p$ are all distinct in $\mathrm{C}_{\mathrm{s}}$ and being relatively prime to $\mathrm{p}$ are each of order $\mathrm{p}^{\alpha}$. Since the order of each element of $K$ divides $p^{i-1}$ and since $i<\alpha$, we have that $S \cap K=\phi$. Hence for any $i, j$ in $S$ we have that the $\operatorname{cosets} K+i, K+j$ are distinct. Let $\mathrm{H}=\mathrm{K} \cup(\mathrm{K}+1) \cup \cdots \cup(\mathrm{K}+(\mathrm{p}-1))$. Then $\mathrm{H}$ is a disjoint union of $\mathrm{p}$ cosets belonging to $\mathrm{K}$ and hence by Theorem 2.1. $\mathrm{H}$ is an unfaithful complex of order $\mathrm{p}^{i}$. Further H must have length $\mathrm{p}$. Otherwise, since by Proposition 2.2. the order of the underlying subgroup $K$ of $H$ must divide the order of the subgroup induced by $H$. This means since $H$ has order $\mathrm{p}^{i}$ and $\mathrm{K}$ has the order $\mathrm{p}^{i-1}$ that $\mathrm{H}$ must have length one. But then since $\mathrm{H}$ contains the zero element of $\mathrm{C}_{\mathrm{S}}$, it must be a subgroup of $\mathrm{C}_{\mathrm{S}}$ of order $\mathrm{p}^{i}$. This is a contradiction since $H$ contains $S$ and each element of $S$ generates $\mathrm{C}_{\mathrm{S}}$.

Lemra 3.6. Let $G=\underset{i=1}{\mathrm{~m}} \mathrm{C}_{\mathrm{s}_{1}}$ be a direct product of cyclic groups $\mathrm{C}_{\mathrm{s}_{1}}$ of order $s_{i}$ where for each $i, I \leq i \leq m, s_{i}=s=p^{\alpha}, p$ a fixed prime and $\alpha$ any number such that $\alpha>1$. Suppose that $m>1$. Then for any $k$, $I \leq k<m$, there exists an unfaithful complex of order $s^{k}$ and an unfaithful complex of order $s^{k} p$ in $G$ each of length $p$.

Proof. Since $m>1$, we can write $G=G_{I} \times C_{s}$, where $G_{I}=\underset{i=1}{m=1} C_{1}$. Case 1. Suppose $v=s^{k}$. Select a subgroup $K$ of $G_{1}$ of order $s^{k-1} p^{\alpha-1}$. This is possible since $I \leq k \leq m-1$ and $\left|G_{1}\right|=s^{m-1}$. Since $\alpha>1$, $K$ is a nonzero subgroup of $G_{1}$. Let $K_{1}=K \times\{0\}$ where we denote by 0 the zero elements of both $G_{1}$ and $C_{S}$. Then $K_{1}$ is a nonzero subgroup of $G$. Let $S=\{(0,0),(0,1), \cdots,(0, p-1)\}$ be the subset of $G=G_{1} \times C_{s}$ where the first component of each element of $S$ is the zero of $G_{1}$. Then, clearly, $K_{I} \cap S$ 
$=\{(0,0)\}$, and since $\alpha>1$, the elements in $S$ are all distinct elements of G. Let $H=K_{1} \cup\left(K_{1}+(0,1)\right) \cup \cdots \cup\left(K_{1}+(0, p)\right)$, that is, a set union of cosets of $K_{1}$ with representatives from $S$. Then by Theorem 2.1., $H$ is an unfaithful complex; since the cosets are pairwise distinct the order of $\mathrm{H}$ is $s^{k}$. Further, $H$ has length $p$. Otherwise, since the underlying subgroup $K_{1}$ of $H$ has order $s^{k-1} p^{\alpha-1}$ and since $H$ contains the zero element of $G, H$ must be a subgroup of order $s^{k}$. This implies that the set $T=\{x / 0 \leq x \leq p-1\}$ consisting of the last components of each element of $H$ is a subgroup of $C_{S}$. This, however, is impossible since each element of $T$ generates the group $C_{S}$ and since $\alpha>1$ the order of $C_{s}$ is larger than $p$. Hence $H$ has order $s^{k}$ and length $p$.

Case 2. Suppose $v=s^{k} p$. Select a subgroup $K$ of $G_{1}$ of order $s^{k}$. This is possible since $I \leq k \leq m-1$. Now proceed exactly as in case $I$ to complete the proof.

Lemma 3.7. Let $G=G_{1} \times G_{2}$ be the direct product of two nonzero finite abelian groups $G_{1}$ and $G_{2}$. Let $K$ be any subgroup of $G_{1}$ and let $H$ be any unfaithful complex of length $\ell$ in $G_{2}$. Let $S$ be the subgroup induced by $H$. Then $H_{1}=K \times H$ is an unfaithful complex of length $\ell$ and the subgroup induced by $\mathrm{H}_{1}$ is $\mathrm{K} \times \mathrm{S}$.

Proof. There exists $a_{1}, a_{2}, \cdots, a_{\ell}$ in $G_{2}$ such that $H=\left(s+a_{1}\right) \cup \cdots$ $U\left(s+a_{\ell}\right)$, a disjoint union according to Theorem 2.1. But, $H_{1}=\bigcup_{i=1}^{l}\left(K \times\left(s+a_{i}\right)\right)$, a disjoint union, and clearly $K \times\left(s+a_{i}\right)=(K \times s)+\left(0, a_{i}\right)$ where 0 denotes the zero of $K$. Hence $\mathrm{H}_{1}=\bigcup_{i=1}^{\ell}\left((K \times S)+\left(0, a_{i}\right)\right)$ and since $K \times S$ is a nonzero subgroup of $G$, we have that $H_{1}$ is an unfaithful complex of $G$. Also $H_{1}+\left(b_{1}, b_{2}\right)$ $=\mathrm{H}_{1}$ if and only if $\mathrm{K}+\mathrm{b}_{1}=\mathrm{K}$ and $\mathrm{H}+\mathrm{b}_{2}=\mathrm{H}$, that is, if and only if $\mathrm{b}_{1} \in \mathrm{K}$ 
and $b_{2} \in S$. Hence $K \times S$ is the subgroup induced by $H_{1}$.

Proposition 3.1. Let $G=\underset{m=1}{t} C_{s}$ be a direct product of cyclic groups $c_{s_{\mathrm{y}}}$ each of order $s=s_{m}$ where for each $m, s=s_{m}=p^{\alpha}, p$ a fixed prime, $t \geq 1$, and $\alpha$ is any number such that $\alpha>1$. Then for each i such that $I<i<\alpha t$ there exists an unfaithful complex of $G$ of order $p^{i}$ and length $p$.

Proof. If $t=1$ the result follows immediately from Lemma 3.5 . Suppose then that $t>1$ and write $G=K \times C_{s}$ where $K=\underset{m=1}{t-1} c_{s_{m}}$. Choose any $i$ such that $1<i<\alpha t$. We can write $i$ in the form $i=k \alpha+j$ for suitable $k$ and $j$ such that $k \geq 0$ and $0 \leq j<\alpha$. Hence we can write $p^{i}=s^{k} p^{j}$ where $j$ and $k$ satisfy the restrictions $k \geq 0,0 \leq j<\alpha$.

Case 1. Suppose that $k=0$. Then since $i>1$, we must have that $j>1$. By Lemma 3.5. there exists an unfaithful complex $H$ of order $p^{j}$ and length $p$ in $\mathrm{C}_{\mathrm{s}}$. Let $\mathrm{K}_{1}=\{0\}$ be the subgroup of $\mathrm{K}$ consisting of the zero element of $K$. Then by Lemma $3.7 \cdot K_{1} \times H$ is an unfaithful complex of $G$ of order $p^{j}$ and length $\mathrm{p}$.

Case 2. Suppose that $j>1$. Then since $i<\alpha t$, it follows that $k \leq t-1$. By Lemma 3.5. there exists an unfaithful complex $H$ of order $\mathrm{p}^{j}$ and length $\mathrm{p}$ in $\mathrm{C}_{\mathrm{s}}$. Let $\mathrm{K}_{1}$ be a subgroup of $\mathrm{K}$ of order $\mathrm{s}^{\mathrm{k}}$. Then by Lemma 3.7 . we have that $K_{1} \times H$ is an unfaithful complex of $G$ of order $p^{i}$ and length $\mathrm{p}$.

Case 3. If $j=0$ or $j=1$, then $p^{i}=s^{k}$ or $p^{i}=s^{k} p$ respectively. Then since $I<i<\alpha t$, we have that $l \leq k<t$ and the result follows in this case from Lemma 3.6. to complete the proof. 
Lemma 3.8. Let $G=\underset{j=I}{\mathrm{~m}} \mathrm{C}_{\mathrm{p}}$, be a direct product of cyclic groups $\mathrm{C}_{p_{j}}$ of order $p=p_{j}$, where for each $j, p_{j}=p$ and $p$ is a fixed prime. Suppose that $m \geq 2$ and let $K$ be any subgroup of $G$ such that $K \neq G$. Then for any $x \notin K$ the cyclic subgroup $S$ of $G$ generated by $x$ is such that $S \cap K=\{0\}$.

Proof. Since each nonzero element of $G$ is of order $p$, it follows that $S$ is a cyclic subgroup of order $p$, so that $S=\{k x / I \leq k \leq p\}$. Suppose now that $K \cap S \neq\{0\}$. Then there exists $y \neq 0$ in $K$ such that $y=k x$ with $1<k \leq p-1$. Hence the g. c. d. $(k, p)=1$ and thus $k x$ is also a generator of the subgroup $S$. Hence for some $k_{1}$ such that, $I \leq k_{1} \leq p$, we have that $x=k_{1}(k x)=k_{1} y$, that is, $x \in K$. This, however, is a contradiction to our choice of $x$; hence the lemma is established.

Proposition 3.2. Let $G={\underset{j=1}{x} \mathrm{p}_{j}}_{\text {be a direct product of cyclic groups }}^{\mathrm{m}}$ $c_{p_{j}}$ each of order $p_{j}=p$ where for each $j, p_{j}=p$ is a fixed prime such that $p \neq 2$. Suppose that $m \geq 3$. Then for each $i$ such that $1<i<m$ there exists an unfaithful complex in $G$ of order $p^{i}$ and length $p$.

Proof. Let $\mathrm{K}$ be any subgroup of $\mathrm{G}$ of order $p^{i-1}$. Since $1<i<m$, it follows that $K$ is a nonzero subgroup and that $K \neq G$. Let $x \in G-K$. By Lemma 3.8. the cyclic subgroup $S$ of $G$ generated by $x$ is such that $K \cap S$ $=\{0\}$; since $p \neq 2$ the subset $\{k x / 0 \leq k \leq p-2\}$ of $s$ consists of distinct elements of $G$. Let $K_{1}=\{y+k x / y \in K, I \leq k \leq p\}$ be the subgroup of $G$ generated by $\mathrm{K} \cup\{\mathrm{x}\}$. Clearly, $\mathrm{K}_{1}$ has order $\mathrm{p}^{i}$, and since $i<\mathrm{m}$, we have that $K_{1} \neq G$. Select an element $\bar{c}$ of $G$ such that $\bar{c} \notin K_{1}$. Let . $H=(K+\ddot{c}) U[U(K+k x) / 0 \leq k \leq p-2\}]$. The cosets whose union is $H$ are clearly distinct, and hence $H$ is an unfaithful complex of $G$ of order $p^{i}$. 
We claim that $H$ has length $p$. Otherwise, since the underlying subgroup $K$ of $H$ has order $\mathrm{p}^{i-1}$ and $H$ coniains the zero element of $G$, it must follow that $H$ is a subgroup of order $p^{i}$. But then since $H$ contains $K$ and $x$, we must have that $\mathrm{H}=\mathrm{K}_{1}$. This, however, is a contradiction since $\bar{c} d K_{1}$. This completes the proof.

Proposition 3.3. Let $G=\underset{j=1}{m} c_{j}$ be a direct product of cyclic groups $\mathrm{C}_{j}$, where for each $j, \mathrm{C}_{j}=\{0,1\}$ is a croljo groun of order two under addition mod. 2 . Then (i) there exists no nonither complexes in G of length two, (ii) if $m \geq 4$ then for each i sing that $2<i<m$ there exists an unfaithful complex of ordex $2^{i}$ and lengtin four, (iii) if $1 \leq \mathrm{m} \leq 3$, then every unfaitiful corplex of $G$ is eiviner of length one, that is, it is a coset belonging to some subgroup of $G$, or it is of length three.

Proof. (i) Suppose that $H$ is an unfaithful complex of $G$ with underlying subgroup $S$ such that $H=\left(S+a_{1}\right) U\left(S+a_{2}\right)$. Since each nonzero element of $G$ is of order two, it follows that the set $T=H+a_{1}=S$ $U\left(S+a_{1}+a_{2}\right)$ is a subgroup of $G$. But then $H=T+a_{1}$ so that $H$ is a coset belonging to the subgroup $T$. It follows that $H$ has length one. (ii) Since $m \geq 4$, there exists at least one $i$ such that $2<i<m$. Pick such an $i$ and let $K$ be a subgroup of $G$ of order $2^{i-2}$. Then since $i>2$, we have that $|K| \neq I$ so that $K$ is a nonzero subgroup of $G$. Also since $i<m$, we have that $K \neq G$. Choose $x \in G-K$ and let $K_{1}$ be the subgroup of $G$ generated by $K \cup\{x\}$, that is, let $K_{1}=\{w+k x / w \in K, 0 \leq k \leq 1\}$. Then clearly $K_{1}$ has order $2^{i-1}$ so that $K_{1} \neq G$. Select $y \in G-K$, and let $K_{2}$ be the subgroup of $G$ generated by $K_{1} \cup\{y\}$. Then $\left|K_{2}\right|=2^{i}$, and since $i<m$, we have that $K_{2} \neq G$. Choose $z \in G-K_{2}$ and let $H=K U(K+x) U(K+y)$ 
$U(K+z)$. Now by construction $K \subset K_{1} \subset K_{2}$ so that the elements $x-y$, $y-z, z-x$ of $G$ do not belong to $K$. Hence the cosets whose union is $H$ are distinct, and the order of $\mathrm{H}$ is $2^{i}$. Now $\mathrm{H}$ must have length four, otherwise, by (i) it cannot have lergin two and hence its length must be one. This means that, $H$ must be a subgroup of order $2^{i}$. Since $H$ contains $\mathrm{x}$ and $\mathrm{y}$, it follows that $\mathrm{H}=\mathrm{K}_{2}$. This, bovrerer, is a contradiction since $z \in H$ but $z \notin \kappa_{2}$. (iii) suppose trat $1 \leq m \leq 3$. If $m=1$ or 2 , it is clear that each uneatheu?. compiex of $G$ has lemgtin $I$. Suppose that $m=3$. By Corollary 2.2. We know thit the omder of ar infeitiful complex $\mathrm{H}$ of $\mathrm{G}$ is then of the form $|\mathrm{H}|=2 \mathrm{k}$ were $I \leq 1 \leq 4$. If $k=1,2$ or 4 , then clearly H must have length one since by (i), vinithrul couplexes of length two do

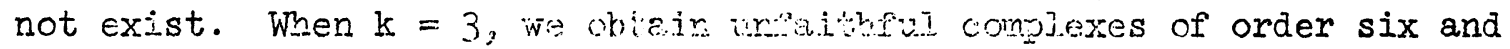
length three in $G$ whose uiderlying sligroups have order two. This completes the proo?

In the next resul's we provide criteria under which formula (3.13) holds when the restriction placed on $\nu$ is removed and we allow $v$ to be any number such that $I \leq v \leq s^{t}$.

Theorem 3.7. Formula (3.13) holds for a given $\nu$ such that $1 \leq \nu \leq s^{t}$ if and only if one of the following conditions is satisfied: (i) the g. $c$. d. $(v, p)=1$, (ii) $v=s^{t},(i i i) v=p$, (iv) $G=c_{2} \times c_{2} \times c_{2}$ and $v \neq 6$ where (a) $s=p^{\alpha}, p$ a fixed prime, $\alpha \geq 1, t \geq 1$ and $(b) c_{2}=\{0,1\}$ is the cyclic group of order two under addition mod. 2 .

Proof. Let $v$ be a number such that $I \leq v \leq s^{t}$. Suppose that formula (3.13) holds, that is, suppose that $\psi_{G}(v) a+f s^{t}=C_{\nu} s^{t}$ where $f=\left|F_{\nu} / \tau\right|$, $a$ is the index of a subgroup of $G$ of order $v$ and $G=C_{s} \times \cdots C_{s}$ ( $t$ factors). 
Then since $f s^{t}=\left|F_{\nu}\right|$ and $c_{\nu}^{s^{i}}=\left|C_{\nu}\right|$, it follows that $\psi_{G}(\nu) a=\left|U_{\nu}\right|$ so that if (3.13) holds then every unfaithful complex of $G$ of order $\nu$ must be a coset belonging to some subgroup of $G$ of order $v$. Thus if (3.13) holds then every unfaithful complex of order $v$ has length one. Now suppose that the conditions (i), (ii) and (iii) fail to hold. We deduce then that (iv) must hold. Suppose then that the g.c. d. $(v, p)=d>I$. Let $v=m p^{i}$ where $\mathrm{p}^{i}$ is the highest power of $p$ dividing $v$. Then the g.c.d. $(m, p)=1$. Suppose now that $m>1$. Let $K$ be any subgroup of $G$ of order $p^{i}$. Since we assume that the condition (ii) fails, we have that $v=m p^{i}<s^{t}=p^{\alpha t}$. Hence the index of $K$ is larger than $m$. Let $H=K \cup\left(K+a_{l}\right) \cup \cdots U\left(K+a_{m-I}\right)$, a union of distinct cosets belonging to $K$. Then $H$ is an unfaithful complex of order $\nu=m p^{i}$. Further $H$ cannot be a subgroup of $G$ since $m>I$ implies that $v$ does not divide the order of $G$. Thus $H$ has length $m>I$ and this is a contradiction to our assumption that (3.13) holds. Hence $m=1$ and as a consequence $v=p^{i}$. Again since $v \neq p, v \neq s^{t}=p^{\alpha t}$ we must have that $I<i<\alpha t$. If $\alpha>1$ then by Proposition 3.1. there exists an unfaithful complex of order $p^{i}$ and length $p$ which would contradict our assumption that (3.13) holds. Thus $\alpha=1$ and $1<i<t$, which means that $t \geq 3$. Again if $p \neq 2$, then by Proposition 3.2. there exists an unfaithful complex of order $\nu=p^{i}$ and length $p$ contradicting our assumption that (3.13) holds. Hence we must have that $p=2$, so that $v=2^{i}, t \geq 3$, and $1<i<t$. Now if $t \geq 4$, then by Proposition 3.3. there exists an unfaithful complex of order $2^{i}$ and length 4 which would contradict our assumption that (3.13) holis. Hence we must have that $t=3$ so that $1<i<t$ implies that $i=2$. This mecns finally tinat $\mathrm{c}_{\mathrm{r}}=\mathrm{C}_{2} \because \mathrm{C}_{2} \times \mathrm{C}_{2}$ and $v=2^{i}=2^{2}$, that is, $v \neq 6$. Fence condition (ir) has beer deduced. Conversely, if conditions (ii) or 
(iii) hold, it is clear that (3.13) holds.. If condition (i) holds, that is, if the g. c. d. $(v, p)=1$, then by Proposition 2.4 we have that $U_{v}=\varnothing$ so that $C_{v}=F_{v}$ and it follows immediately that (3.13) holds. Finally suppose that $G=C_{2} \times C_{2} \times C_{2}$ and $v \neq 6$. Then $|G|=8$ so that if $v$ is any number such that $I \leq v \leq 8$ and the g. c. d. $(v, 2)=1$, then again by Proposition 2.4. $U_{v}=\phi$ so that $F_{v}=C_{v}$ and $(3.13)$ holds. If $v=2$ then all unfaithful complexes of $\mathrm{G}$ of order two are of Iength 1 and if $\nu=8$, then $F_{\nu}=\phi$ so that in either case (3.13) holds. Finally if $v=4$, then again by Proposition 3.3. all unfaithful complexes of $G$ of order four are of length one and the formula (3.13) holds. This completes the proof.

4. Application to fractional factorial designs. In this section we show how the theory developed in the previous two sections can be used to classify and enumerate designs. The main objectives of this section are the following: (i) to show how designs may be identified with the concepts of faithful and unfaithful complexes and to use this idea to exhibit a method for the construction and enumeration of a class of designs and (ii) to describe a classification problem for a class of designs and then to show, in general, to what extent the theory developed in the previous two sections helps towards a resolution of this problem. We conclude with a practical example to illustrate the ideas mentioned in (i) and (ii) above.

We now provide the basic setting in which the ideas of this section will be formulated.

Let $G=\underset{i=1}{m} C_{s_{1}}$ be a direct product of groups where for each $i, I \leq i \leq m$, $c_{s_{1}}=\left\{0,1,2, \cdots, s_{i}-1\right\}$ is a cyclic group under addition mod. $s_{i}$ and let $\mathrm{n}=\pi \mathrm{s}_{\mathrm{i}}$. We call the elements of $\mathrm{G}$ treatments and by a design $D$ of $\mathrm{G}$ we 
mean a collection of treatments of $G$ where it is understood that any given treatment in $D$ may possibly, though not necessarily, be repeated more than once. With each design $D$ of $G$ we associate a complex $\bar{D}$ of $G$ consisting of the set of distinct treatments in $D$. We call $\bar{D}$ the underlying complex of the design $D$. It is clear under these definitions that any complex of $G$ is a design and further that any given complex $H$ of $G$ generates an infinite class $0:$ designs $D$ whose underlying complex is $H$. It is also clear that the class of designs $D$ with underlying complex $H$ is known once $H$ is given. We say that a design $D$ has distinct order $v, I \leq \nu \leq n$, if and only if the underlying complex $\bar{D}$ of $D$ has order $\nu$. It now follows from the foregoing that the construction and enumeration of the class of designs of distinct order $\nu$ reduces to the study of the set $C_{\nu}$ consisting of the complexes of $G$ of order $v$. The study of the set $C_{\nu}$ of complexes of order $v$ in $G$ can be further reduced by the consideration of a subset $T$ of $C_{v}$ consisting of complexes of order $v$ one from each equivalence class in the quotient set $C_{v / \tau}$. Such a set $J$, as remarked earlier is characterized by the properties that (a) $\tilde{\Omega}(G) * \mathcal{J}=C_{v}$ and $(b)$ if $\& \subseteq C_{v}$ such that $\tilde{\Omega}(G) * s=C_{v}$ then $|J| \leq|s|$.

The following two general remarks can be made: (a) recall that $C_{\nu}$ $=F_{v} \cup U_{v}$ a disjoint union of the set $F_{v}$ of faithful complexes of order $v$ and the set $U_{v}$ of unfaithful complexes of order $v$. Thus, clearly, we can write the set $J$ mentioned above as a disjoint union $J=J_{0} \cup \bar{J}_{0}$ where $\tau_{0} \subseteq U_{v}$ and consists of unfaithful complexes of order $v$ one from each equivalence class of the quotient set $U_{v} / \tau$, and, $\bar{\tau}_{0} \subseteq F_{v}$ and consists of faithful complexes of order $\nu$ one from each equivalence class of the quotient set $F_{\nu / \tau}$, (b) in constructing the subsets $J_{0}$ and $\bar{J}_{0}$ of $U_{\nu}$ and $F_{\nu}$ respectively, it is clearly enough to list complexes in $J_{0}$ and $\bar{J}_{0}$ respectively which contain the zero element of $G$. 
Let $v$ be any number such that $1 \leqslant v: \leq n$. Then the set $C_{v}$ has cardinality $c_{v}^{n}$ and in the light of the previous two sections the construction of the sets $J_{0}$ and $\bar{J}_{0}$ mentioned above may be considered under the following two cases depending on the number $v$.

Case 1. Suppose that the g.c.d. $(v, n)=1$. Then by Proposition 2.4. there are no unfaithful complexes of order $v$ so that $U_{v}=\phi$ and $C_{\nu}=F_{\nu}$. Let $f=\left|F_{\nu} / \tau\right|$. Select faithful complexes one from each equivalence class in $\mathrm{F}_{\nu / \tau}$ and let $\bar{J}_{0}=\left\{\mathrm{H}_{1}, \mathrm{H}_{2}, \cdots, \mathrm{H}_{f}\right\}$ be the resulting set. Then clearly every complex $\mathrm{H}$ in $\mathrm{C}_{\nu}$ is of the form $\mathrm{H}=\mathrm{H}_{i}+\mathrm{h}$ for exactly one $h$ in $G$ and exactly one $i$ such that $l \leq i \leq f$. Finally by Corollary 3.j. the number $f=\left|F_{v / \tau}\right|$ is given by $f=\frac{1}{v} C_{\nu-1}^{n-1}$ if $v \geq 2$ and $f=1$ if $v=1$.

Case 2. Suppose now that the g. c. $d \cdot(\nu, n)=d>1$. We first construct the class $U_{v}$ of unfaithful complexes of order $v$. Let $D$ $=\left\{a_{1}, d_{2}, \cdots, d_{t}\right\}$ be the set given in (2.2), namely, the set of all divisors of $d$ distinct from one and suppose that $d_{1}<d_{2}<\cdots<d_{t}$. Let $v=k d, d=m_{i} d_{i}, r_{i}=\frac{n}{d_{r}}$ and $b_{i}=k m_{i}$ where $I \leq i \leq t$ : Then by Proposition 2.3. the various possible lengths for unfaithful complexes of order $v$ is in the set $L=\left\{b_{1}, b_{2}, \cdots, b_{t}\right\}$ defined in (2.3). Note that $b_{1}>b_{2}>\cdots>b_{t}$. Also by Lemma 3.2. $U_{v}=U\left\{x_{b_{1}}\left(K_{d_{1}}\right) / I \leq i \leq t\right\}$, where the sets $\mathrm{x}_{\mathrm{b}_{1}}\left(\mathrm{~K}_{\mathrm{d}_{1}}\right)$, consisting of unfaithful complexes of length $b_{i}$ and induced subgroup of order $d_{i}$, are pairwise disjoint. Thus to build the set $U_{v}$ it is enough to restrict ourselves to constructing the set $x_{b_{1}}\left(K_{d_{1}}\right)$. Let $I \leq i \leq t$ and recall that $c_{i}=\left|x_{b_{1}}\left(K_{d_{1}}\right) / \tau\right|$. We begin by listing the set $K_{d_{i}}$ of subgroups of $G$ of order $d_{i}$. Next list all those 
unfaithful complexes $H$ of $G$ which are unions of $b_{i}$ distinct cosets belonging to some subgroup in the set $K_{d_{1}}$. This is precisely the set of all unfaithful complexes of $G$ of order $\nu$ whose underlying subgroup belongs to the set $\mathrm{K}_{\mathrm{d}_{1}}$. Reduce this set by removing those unfaithful complexes in it which have length smaller than $b_{i}$. From the resulting set which is $x_{b_{1}}\left(K_{d_{1}}\right)$, construct the set $I_{i}$ of cardinality $c_{i}$, where by Theorem $3 \cdot 3$. $c_{i}=\frac{l}{r_{1}}\left|x_{b_{1}}\left(K_{d_{1}}\right)\right|$, consisting of unfaithful complexes of order $v$ one from each equivalence class in the quotient set $x_{z_{1}}\left(K_{\tilde{a}_{i}}\right) / \tau$. Let $I_{0}=\bigcup_{i=I}^{t} J_{i}$, then $I_{0}$ has cardinality $u=\sum_{i=I}^{t} c_{i}$ and the set $I_{0}$ $=\left\{\mathrm{H}_{1}, \mathrm{H}_{2}, \cdots, \mathrm{H}_{\mathrm{u}}\right\}$ generates $U_{v}$ under the action of the group $\tilde{\Omega}(\mathrm{G})$. Next consider the set $C_{v}-U_{v}=F_{v}$ and let $\bar{J}_{0}=\left\{H_{l}^{\prime}, H_{2}^{\prime}, \cdots, H_{f}^{\prime}\right\}$ be a set of faithful complexes one erom each equivalence ciass in the quotient set $F_{\nu / \tau}$ where $f=\left|F_{v} / \tau\right|$. Fincily the set $\tau=\tau_{0} U \bar{\tau}_{0}$ consisting of $c=f+u$ complexes of order $v$ is the set with least cardinality such that the action of the group $\tilde{\Omega}(G)$ upon it generaties the set $C_{v}$.

In addition let $\bar{T}$ be the set coinsisting of the complexes of order $n-v$ which are set complements in $G$ of the complexes in the set $I$ mentioned above. Then according to Proposition 2.4. We must have that the set $\overline{\mathcal{J}}$ is the subset of $C_{n-v}$ of smallest cardinality which generates the set $C_{n-v}$ under the action of the group $\tilde{\Omega}(G)$. Thus the enumeration and construction of the set $\mathcal{I}$ which generates $C_{\nu}$ under the action of the group $\tilde{\Omega}(G)$ also leads to the construction of the set $\bar{J}$ which generates the set $C_{n-\nu}$ under the action of the group $\tilde{\Omega}(G)$, and conversely.

Let $v$ be any number such that $I \leq v \leq n$. Let $H$ be a complex of order $v$, and let $\left(r_{h}\right)_{h \in H}$ be any sequence of positive numbers. We say that the 
design $D$ of $G$ is obtained by replication from the pair $\left(H,\left(r_{h}\right)_{h \in H}\right)$ if and only if the underlying complex of $D_{\Omega}$ is $H$ and each treatment $h$ in $H$ is repeated exactly $r_{h}$ times. In this case we write $D=\left(H,\left(r_{h}\right)_{h \in H}\right)$. It is now clear that each design $D$ of $G$ of distinct order $v$ is of the form $D=\left(\bar{D},\left(r_{\overline{\mathrm{d}}}\right)_{\tilde{\mathrm{d}}_{\in} \overline{\mathrm{D}}}\right)$ and conversely given a pair $\left(\mathrm{H},\left(\mathrm{r}_{\mathrm{h}}\right)_{\mathrm{h} \in \mathrm{H}}\right)$, where $\mathrm{H}$ is a complex of order $v$, there exists exactly one design $D$ of distinct order $v$ such that $D=\left(H,\left(r_{h}\right)_{h \in H}\right)$. The process of obtaining the unique design $D$ from the pair $\left(H,\left(r_{h}\right)_{h \in H}\right)$, we will call the renlication operation. Note, in particular, that if $H$ is any complex of order $v$, then $H=\left(H,\left(r_{h}\right)_{h \in H}\right)$ with $r_{h}=1$ for each $h \in H$.

In terms of the replication operation we may summarize the above ideas in the following way. Let $\Delta$ be the class of all designs of $G$ and for each $\nu, I \leq \nu \leq n$, let $A$, be the class of all designs of $G$ of distinct order $v$. Then, clearly, $\Delta=U\{\Delta / I \leq \nu \leq n\}$ and this set union is pairwise disjoint. It follows then that the study of the set $\Delta$ reduces to the study of each of the sets $\Delta_{\nu}$. From the above we have seen that the study of the class of designs of distinct order $v$ may be reduced to the consideration of the set $J_{\nu}$ of complexes of order $v$ which has least cardinality with respect to the property that $\tilde{\Omega}(G) * J_{v}=C_{v}$. Further, the number $c$ which is the cardinality of the quotient set $C_{v} / \tau$ is given by formula (3.9) of Corallary 3.2. Now, let $D$ be any design in $\Delta$. Then, $D$ belongs to $\Delta$ for exactly one $v$ such that $I \leq v \leq n$, and $D$ is obtained by replication from its underlying complex $\bar{D}$. Of course, $\bar{D}$ belongs to $C_{v}$ and hence, there exists exactly one $H$ in $J_{V}$ and exactly one $h$ in $G$ such that $\bar{D}=H+h$, that is, $\bar{D}$ is obtained by translation from a unique complex in $J_{v}$. Hence, we have established that the class of all designs $\Delta$ of $G$ is generated by the operation 
of translation followed by the operation of replication on the set $\bigcup_{v=1}^{n} g_{v}$ of complexes of $G$.

Let $D_{1}$ and $D_{2}$ be any two designs in $\Delta_{v}$. Let $p$ be a fixed set of parameters and let $M_{D_{1}, p}, M_{7}, p$ be the information matrices of $D_{1}$ and $D_{2}$ respectively with respect to $p$. We say that $D_{1}$ is similar to $D_{2}$ with respect to $p$, in symbols, $D_{1} \cong D_{2}$ if and only if the information matrices $M_{1}, p$ and ${ }_{D_{B}, p}$ have the same spectra. A general classification problem for the designs in $\Delta_{v}$ would be to obtain a description of the equivalence class $\left[D \cong_{0}\right.$ of the quotient set $\Delta_{\nu} / \cong_{p}$ consisting of all designs similar to $D$ with respect to $\mathrm{p}$. While no such description is available at the moment, the theory developed so far combined with a result in a recent paper by Srivasta, Raktoe and Pesotan [1971] helps in giving a description for some of the members, though not necessarily all, of the equivalence class $[D] \cong_{\mathrm{F}}$ with respect to a suitable choice of parameters $p$.

For each $j, l \leq j \leq m$, let $\omega_{j}$ be a permutation on the underlying set of the cyclic group $C_{s_{j}}$ of order $s_{j}$ and consider the group $\Omega(G)$, introduced in general in (2.9), consisting of permutations $\omega=\left(\omega_{1}, \cdots, \omega_{m}\right)$ on $G$ defined by $\omega\left(b_{1}, \cdots, b_{m}\right)=\left(w_{1}\left(b_{1}\right), \cdots, w_{m}\left(b_{m}\right)\right)$ for any $\left(b_{1}, \cdots, b_{m}\right)$ in $G$. For any complex $H \cong G$ let $\omega(H)=\{\omega(h) / h \in H\}$ and let a design $D$ in $\Delta_{V}$ be given. Suppose that $D=\left(\bar{D},\left(r_{\bar{d}}\right)_{\bar{d} \in \bar{D}}\right)$ where $\bar{D}$ is the underlying complex of $D$. We now define

(4.I) $\quad \Omega(G) * D=\left\{D_{1} / D_{1} \in \Delta_{v}, D_{1}=\left(w(\bar{D}),\left(r_{w(\bar{d})}\right)_{\bar{d} \in \bar{D}}\right)\right.$ and $\left.\omega \in \Omega(G)\right\}$ We call the set $\Omega(G) * D$, the set of designs of distinct arder $v$ generated by the action of the group $\Omega(G)$ an the design $D$ in $\Delta_{v}$. It is now clear that by 
the replication operation applied in turn to each nember of the set $\Omega(G) * \bar{D}$ one obtains the set $\Omega(G) * D$. Hence the study of the set $\Omega(G) * D$ reduces to the study of the set $\Omega(G) * \bar{D}$ where $\bar{D}$ is the underlying complex of $D$. Now, $\Omega(G) * \bar{D}$ is a subset of $C_{v}$. Hence uising the fact that $\tilde{\Omega}(G) * J_{\nu}=C_{v}$ and Theorem 2.5. we know that there exists a set of complexes $\&_{\bar{D}}$, depending on $\bar{D}$, contained in $J_{v}$ such that $\tilde{\Omega}(G) * \delta_{\bar{D}}=\Omega(G) * \bar{D}$. Thus we have established that for each $D \in \mathcal{Q}_{v}$ there exists a set $\Omega_{\bar{D}} \subseteq J_{\nu}$ such that $\tilde{\Omega}(G) * \delta_{\bar{D}}=\Omega(G) * \bar{D}$. Now, suppose that $J_{v}=\left\{H_{1}, H_{2}, \cdots, H_{c}\right\}$; let $\bar{J}_{v}=\left\{H_{i_{1}}, \ldots, H_{i_{k}}\right\}$ be a subset of $I_{\nu}$ of least cardinality such that $\Omega(G) * \bar{J}_{\nu}=C_{\nu}$. Then, by the foregoing, for each $j, I \leq j \leq k$, we have that there exists a set $\varepsilon_{H_{j}} \subseteq J_{\nu}$ such that $\Omega(G) * H_{i_{j}}=\tilde{\Omega}(G) * \&_{H_{j}} \cdots$ It follows that

$$
c_{\nu}=\Omega(G) * \bar{J}_{\nu}=\left(\tilde{\Omega}(G) * \varepsilon_{H_{i_{1}}}\right) \cup \cdots \cup\left(\tilde{\Omega}(G) * \beta_{\mathrm{H}_{i_{*}}}\right)
$$

and this union is clearly pairwise disjoint. Further, we have that $\mathcal{T}_{\nu}$ $=\&_{\mathrm{H}_{i_{1}}} U \cdots \cup \varepsilon_{\mathrm{H}_{i_{k}}}$ a pairwise disjoint union. The importance of the decomposition of the set $C_{\nu}$ of complexes of order $\nu$ as a disjoint union of the sets $\tilde{\Omega}(G) * s_{\mathrm{H}_{i_{j}}}, I \leq j \leq k$, depends on the result obtained recently in a paper by Srivasta, Raktoe and Pesotan [1971]. We give here a brief summary of this result.

-..met us associate with each of the cyclic groups $C_{s_{1}}$ a formal symbol $A_{i}$ and with each treatment combination $\left(i_{1}, i_{2}, \ldots, i_{m}\right)$ in $G$ let us associate the formal symbol $A_{1}^{i_{1}} A_{2}^{i_{2}} \ldots A_{m}^{i_{m}}$ which we call an effect and a formal symbol $Y_{i_{1}} \ldots i_{m}$ which we call an observation. With any design $D$ of $G$ we associate an observation vector $Y_{D}$ which is a column vector whose entries are the 
observations $Y_{i_{1}} \ldots i_{m}$ with $\left(i_{1}, \ldots, i_{m}\right)$ in $D$ arranged in some arbitrary order. Let $\mathrm{p}_{0}$ be the set of all effecț, We place the lexicographic order on $G$ and induce it on $p_{0}$, that is, we define $A_{1}^{i_{1}} A_{2}^{i_{2}} \cdots A_{m}^{i_{m}}<A_{1}^{j_{1}} A_{2}^{j_{2}} \cdots A_{m}^{j_{m}}$ if and only if for the first $l, I \leq l \leq \mathrm{m}$ such that $i_{2} \neq j_{l}$ we have that $i_{\ell}<j_{\ell}$. If $p$ is any nonempty set of effects, we denote by $\underline{p}$ the column vector obtained by arranging the effects in $p$ in the order defined on $p_{0}$. Let $\mathrm{X}=\mathrm{N}_{1} \otimes \ldots \otimes \mathrm{N}_{\mathrm{m}}$ be the Kronecker product of matrices $\mathrm{N}_{1}, \mathrm{~N}_{2}, \ldots, \mathrm{N}_{\mathrm{m}}$ where for each $i, I \leq i \leq m, N_{i}$ satisfy the following requirements: (a) $N_{i}$ is a real orthogonal $s_{i} \times s_{i}$ matrix, (b) the first column of $N_{i}$ has the same entry, namely $\frac{1}{\sqrt{s_{1}}}$, (c) the sum of all terms in any column of $N_{i}$ but the first is zero. Further, let $\tau: G \rightarrow\{1,2, \cdots, n\}$ be a map given by $\tau\left(k_{1}, \cdots, k_{m}\right)$ $=j$ if and only if $\left(k_{1}, \ldots, k_{m}\right)$ is the $j^{t h}$ element of $G$ in the lexicographic order in $G$.

Let $D$ be a given design of $G$ and $\underline{p}$ a column vector of effects. Suppose that the observation vector $\mathrm{Y}_{D}$ is displayed as

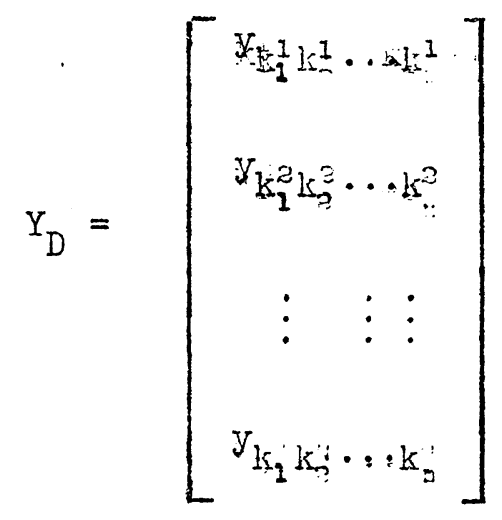

Then we define for any permutation $\omega$ in $\Omega(G)$ a new observation vector determined by $D$ and $\omega$ as 
$(4.4)$

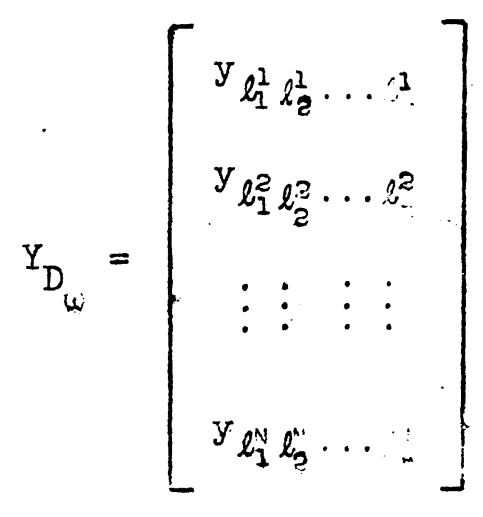

where $\left(l_{1}^{j}, \cdots, l_{m}^{j}\right)=\left(\omega_{1}\left(k_{1}^{j}\right), \cdots, \omega_{m}\left(k_{m}^{j}\right)\right)$. for $1 \leq j \leq N$ and $\omega=\left(\omega_{1}, \cdots, \omega_{m}\right)$.

Further, suppose that the column vector of effects $p$ is displayed as below:

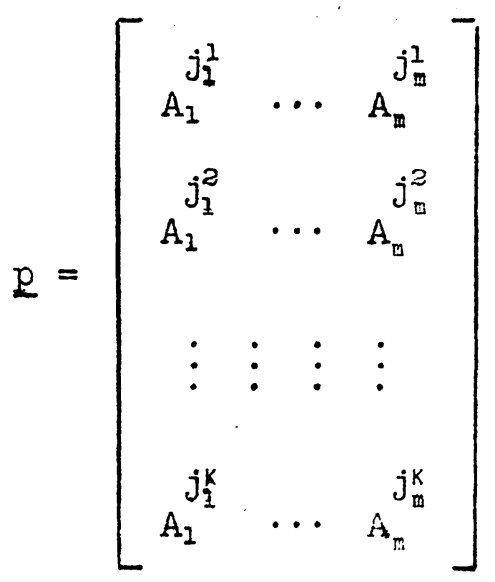

We now define the matrix $X_{D, p}$ as a $N \times K$ submatrix of $X$ in the following way:

Corresponding to the entry $y_{k_{1}^{l} \cdots k_{r}^{1}}$ in $Y_{D}$ and $A_{1}^{j_{2}^{l}} A_{3}^{j_{2}^{l}} \cdots A_{m}^{j_{y}^{l}}$ in $\underline{p}$ with $\tau\left(k_{1}^{1}, \cdots, k_{m}^{1}\right)=n_{i}, I \leq i \leq \mathbb{N}$, and $\tau\left(j_{1}^{l}, \cdots, j_{m}^{l}\right)=t_{\ell}, I \leq l \leq K$, define the $(i, l)$-th entry of $x_{D, p}$ as the $\left(n_{i}, t_{l}\right)$-th entry of $X$.

For any design $D$ and a given column vector of effects $p$ we call the matrix $M_{D, p}=X_{D, p}^{\prime} X_{D, p}$ the information matrix of $D$ with respect to the set of effects $p$ where for any matrix $A$ the matrix $A^{\prime}$ is the transpose of $A$. 
A collection of effects $p$ will be called allowable if and only if whenever $A_{1}^{i_{1}} A_{2}^{i_{2}} \ldots A_{m}^{i_{m}}$ belongs to $p$ and $i_{j} \neq 0,1 \leq j \leq m$, then $A_{1}^{i_{2}} \ldots A_{j-1}^{l_{j-1}} A_{j}^{\ell} A_{j+1}^{i_{j+1}} \ldots A_{m}^{i_{m}}$ belongs to $p$ for all $\ell \neq 0$ in $C_{s_{j}}$. In the paper by Srivastava, Raktoe and Pesotan [1971] the term "admissible" was used in place of allowable. We have abandoned the use of the former term here, since the term admissible has special meaning in statistics.

In the paper by Srivastava, Raktoe and Pesotan [1971] the following theorem was established:

Theorem: Let $Y_{D}$ be an observation vector associated with a design $D$ and let $p$ be a column vector associated with an allowable set of effects $p$. Then each matrix in the set $\left\{\mathrm{M}_{\omega}, \mathrm{p} / \omega \in \Omega(G)\right\}$ of information matrices generated by the design $D$ and $\omega$ in $\Omega(G)$ has the same spectra.

Applying the above theorem we observe that if $\mathrm{p}$ is any allowable set of effects and $D$ is any design in $\Delta_{\nu}$ then the set $\Omega(G) * D$ defined in $(4.1)$ is such that $\Omega(G) * D \subseteq[D]_{\cong p}$, the equivalence class in the quotient set $\Delta_{\nu / \cong p}$ determined by $D$ and $p$. Further, applying this theorem to the decomposition of $C_{\nu}$ given in (4.2) we observe that for each $j, I \leq j \leq k$, the information matrices corresponding to the designs in the set $\tilde{\Omega}(G){ }^{*}{ }_{H_{j}}$ with respect to an allowable set of effects $p$ have the same spectra.

The following example illustrates some of the ideas mentioned above.

Example. Let $\mathrm{G}=\mathrm{C}_{2} \times \mathrm{C}_{3} \times \mathrm{C}_{3}$ be a direct product of cyclic groups where $C_{2}=\{0,1\}$ is a cyclic group of order 2 under addition mod. 2 and 
$C_{3}=\{0,1,2\}$ is a cyclic group of order 3 under addition mod. 3 . For a saturated main effect plan we need to take $\nu=(2-1)+(3-1)+(3-1)+1=6$ treatments of $G$. Then since $|G|=18$ we have that the g.c.d. $(6,18)=d=6$. The divisors of 6 distinct from 1 are then $d_{1}=2, a_{2}=3, a_{3}=6$ and the various possible lengths for unfaithful complexes of order 6, using Proposition 2.3., are given by $b_{1}=3, b_{2}=2, b_{3}=1$. Now the cardinality of the set of complexes of $G$ of order $\nu=6$ is $\left|c_{\nu}\right|=\left|c_{6}\right|=c_{6}^{18}=18,564$. Using formula (3.10) we see that the number of subgroups of $G$ of orders 2,3 and 6 , respectively, are $\psi_{G}(2)=1, \psi_{G}(3)=4$ and $\psi_{G}(6)=4$. Using formula (3.12) of Theorem 3.6. we now obtain the following: (i) the number of unfaithful complexes of order 6 and length 1, namely, $\left|\mathrm{x}_{1}\left(\mathrm{~K}_{6}\right)\right|=\mid \mathrm{x}_{\mathrm{b}_{3}}\left(\mathrm{~K}_{\mathrm{d}_{3}}\right) \dot{i}:=\left(\psi_{\mathrm{G}}(6)\right) \mathrm{c}_{1}^{3}=12$. (ii) the number of unfaithful complexes of order 6 and length 2 , namely, $\left|\mathrm{x}_{2}\left(\mathrm{~K}_{3}\right)\right|=\left|\mathrm{x}_{\mathrm{b}_{2}}\left(\mathrm{~K}_{\mathrm{d}_{2}}\right)\right|=\left(\psi_{G}(3)\right) \mathrm{c}_{2}^{6}$ - (I2) $(1)=48$. (iii) The number of unfaithful complexes of order 6 and length 3 , namely, $\left|x_{3}\left(K_{d_{2}}\right)\right|=\left|x_{b_{1}}\left(K_{d_{1}}\right)\right|=\left(\psi_{G}(2)\right) c_{3}^{9}-(0)(48)-(1)(12)=72$. Hence the number of unfaithful complexes of order 6 , namely, $\left|U_{6}\right|=\sum_{i=1}^{3}\left|x_{b_{1}}\left(K_{d_{i}}\right)\right|$ $=132$ and by Corollary 3.1. we have that $\left|U_{6 / \tau}\right|=20$. Further using formula (3.8) we have that the cardinality of the quotient set $\left|F_{6 / \tau}\right|$ $=\frac{\mathrm{C}_{6}^{18}-132}{18}=1024$. Let $\mathrm{J}_{6} \subseteq \mathrm{C}_{6}$ be a set consisting of complexes of order 6 one from each equivalence class of the quotient set $\mathrm{C}_{6 / \tau}$. Then by formula (3.9) we know that $\left|g_{6}\right|=1024+20=1044$. Thus the 1044 complexes of order 6 in $\tau_{6}$ will generate under translation the 18,564 complexes in $\mathrm{C}_{6}$ and then furtiner by replication all the designs of $G$ of distinct order 6 . Let us write $I_{5}=J_{6}^{0} \cup \tau_{6}^{\prime}$ where $J_{6}^{0}$ consists of all unfaithful complexes of order 6 and $\tau_{6}$ is the remaining complexes in $J_{6}$ which must necessarily be 
faithful. Then from the above $\left|J_{6}^{0}\right|=20$. and $\left|J_{6}^{2}\right|=1024$. We follow the procedure outlined earlier in this section to give a listing of the 20 unfaithful complexes in $\sigma_{6}^{\circ}$. There is one subgroup of order two in $G$, namely, $S=\{(0,0,0),(1,0,0)\}$. There are four subgroups of order three, namely, $s_{1}=\{(0,0,0),(0,1,0),(0,2,0)\}, s_{2}=\{(0,0,0),(0,0,1),(0,0,2)\}$, $S_{3}=\{(0,0,0),(0,1,1),(0,2,2)\}$, and $s_{4}=\{(0,0,0),(0,1,2),(0,2,1)\}$. Finally, there are four subgroups of order six, namely,

$$
\begin{aligned}
& \mathrm{T}_{1}=\{(0,0,0),(0,1,0),(0,2,0),(1,0,0),(1,1,0),(1,2,0)\}, \\
& \mathrm{T}_{2}=\{(0,0,0),(1,0,1),(1,0,2),(1,0,0),(0,0,1),(0,0,2)\}, \\
& \mathrm{T}_{3}=\{(0,0,0),(0,1,1),(0,2,2),(1,0,0),(1,1,1),(1,2,2)\}, \\
& \mathrm{T}_{4}=\{(0,0,0),(0,1,2),(0,2,1),(1,0,0),(1,1,2),(1,2,1)\} .
\end{aligned}
$$

Then the set $\left\{{ }_{1}, \mathrm{~T}_{2}, \mathrm{~T}_{3}, \mathrm{~T}_{4}\right\}$ is a set of complexes of order 6 and length $I$ which generates the set $x_{b_{3}}\left(K_{d_{3}}\right)=x_{1}\left(K_{6}\right)$ under the action of the group $\tilde{\Omega}(G)$. We now list 8 unfaithiul complexes of order 6 and length 2 with induced subgroups of order? which generate the set $\mathrm{X}_{\mathrm{b}_{2}}\left(\mathrm{~K}_{\mathrm{d}_{2}}\right)=\mathrm{X}_{2}\left(\mathrm{~K}_{3}\right)$ under the action of the $\operatorname{scoup} \hat{\Omega}(G)$. These are,

$$
\begin{aligned}
& T_{5}=\{(0,0,0),(0,1,0),(0,2,0),(0,0,1),(0,1,1),(0,2,1)\}, \\
& T_{6}=\{(0,0,0),(0,1,0),(0,2,0),(0,1,2),(0,2,2),(0,0,2)\}, \\
& T_{7}=\{(0,0,0),(0,0,1),(0,0,2),(0,1,2),(0,1,0),(0,1,1)\}, \\
& T_{8}=\{(0,0,0),(0,0,1),(0,0,2),(0,2,1),(0,2,2),(0,2,0)\}, \\
& T_{9}=\{(0,0,0),(0,1,2),(0,2,1),(0,0,1),(0,1,0),(0,2,2)\}, \\
& T_{10}=\{(0,0,0),(0,1,2),(0,2,1),(0,1,1),(0,2,0),(0,0,2)\} \\
& T_{11}=\{(0,0,0),(0,1,1),(0,2,2),(0,1,2),(0,2,0),(0,0,1)\}
\end{aligned}
$$




$$
T_{12}=\{(0,0,0),(0,1,1),(0,2,2),(0,2,1),(0,0,2),(0,1,0)\}
$$

Finally we list 8 unfaithful complexes of order 6 and length 3 which generate the set $x_{b_{1}}\left(K_{d_{1}}\right)=x_{3}\left(K_{2}\right)$ under the action of the group $\tilde{r}(G)$. These are,

$$
\begin{aligned}
& T_{13}=\{(0,0,0),(1,0,0),(1,0,2),(0,0,2),(1,2,0),(0,2,0)\} \\
& T_{14}=\{(0,0,0),(1,0,0),(1,0,2),(0,0,2),(0,1,0),(1,1,0)\} \\
& T_{15}=\{(0,0,0),(1,0,0),(1,0,2),(0,0,2),(1,2,1),(0,2,1)\} \\
& T_{16}=\{(0,0,0),(1,0,0),(1,0,2),(0,0,2),(1,2,2),(0,2,2)\} \\
& T_{17}=\{(0,0,0),(1,0,0),(1,0,1),(0,0,1),(1,2,0),(0,2,0)\} \\
& T_{18}=\{(0,0,0),(1,0,0),(1,0,1),(0,0,1),(0,1,0),(1,1,0)\} \\
& T_{19}=\{(0,0,0),(1,0,0),(1,0,1),(0,0,1),(1,2,1),(0,2,1)\} \\
& T_{20}=\{(0,0,(),(1,0,0),(1,0,1),(0,0,1),(1,2,2),(0,2,2)\}
\end{aligned}
$$

Hence the set $J_{6}^{0}=\left\{T_{i} / 1 \leqslant i \leqslant 20\right\}$ will generate the set of all unfaithful complexes of order six under the action of the group $\tilde{\Omega}(G)$. Further, to obtain a comlete 1 istirg of the set $J_{6}$ one would have to list 1024 faithful complexes of wrlec 6 which are not related by a translation; each such faithful complex of order 6 in $J_{6}$ will produce 18 complexes under translation. Since we selected the parameters $p$ to be the main effects and the mean then, clecrly, $p$ is an allowable set of effects. Thus if $D$ is any design of distinct orcier 6 , then the class of designs generated by the action of the group $\Omega(G)$ on $D$, naraly the class $\Omega(G) * D$, is spectra invariant with respect to the set of main effects $p$. Also, in the list $J_{6}$ of 1044 faithful and unfaithful complexes if we select a complex $H$ and obtain through it a design $D=\left(H_{0},\left(r_{h}\right)_{h \in H}\right)$, then the class of designs generated by the action of $\tilde{\Omega}(G)$ on $D$ is as well spectrum invariant relative to the set of 
main effects $p$. Finally, one might measure the efficiency of this process by observing that we have discarded 17,520 complexes of order 6 so that this process has a discarding efficiency of $94.2 \%$ in this case.

\section{REFERENCES}

[1] BOSE, R. C. (1947). Mathematical theory of the symmetrical factorial design. Sankhyā 8 107-166.

[2] CARMICHAEL, R. D. (1956). Introduction to the theory of groups of finite order. Dover Publications, New York.

[3] FISHER, R. A. (1942). The theory of confounding in factorial experiments in relation to the theory of groups. Ann. Eigenics 11 341-353.

[4] PAIK, U. B. and FEDIRIR, W. T. (1970). A randomized procedure of saturated main effsct fractional replicates. Ann; Math. Statist. 41 369-375.

[F] RAITOF, B. L. and FEDERER, W. T. (1971). On the number of generators of siburatej min effect plans. Ann. Math. Etesiat. 42 1758-1760.

[5] SRIVASTAVA, J. N., RAKTOI, B. I. and PE:ONAN, H. (1971). On invariance and randomization in fractional replication. (Paper in the process of publication.) 